\title{
World-volume effective theories of locally non-geometric branes
}

\author{
Kenta Shiozawa and Shin Sasaki \\ Department of Physics, Kitasato University, \\ Sagamihara 252-0373, Japan \\ E-mail: k.shiozawa@sci.kitasato-u.ac.jp, shin-s@kitasato-u.ac.jp
}

ABSTRACT: We study world-volume effective theories of five-branes in type II string theories. We determine the bosonic zero-modes of the NS5-brane, the Kaluza-Klein monopole, the exotic Q5-, R5-branes and a space-filling brane, by direct calculations within the formalism of double field theory (DFT). We show that these zero-modes are Nambu-Goldstone modes associated with the spontaneously broken gauge symmetries in DFT. They are organized into the bosonic part of the six-dimensional $\mathcal{N}=(1,1)$ vector and the $\mathcal{N}=(2,0)$ tensor multiplets. Among other things, we examine the locally non-geometric R5-branes and space-filling branes that are characterized by the winding space. We also study effective theories of five-branes with string worldsheet instanton corrections.

Keywords: p-branes, String Duality, Brane Dynamics in Gauge Theories

ARXiv EPrint: 2010.02701 


\section{Contents}

1 Introduction 1

2 Locally non-geometric branes in double field theory 3

3 Translational zero-modes in doubled space $\quad 9$

$\begin{array}{ll}3.1 \text { Zero-modes equation } \delta \mathcal{R}_{M N}=0 & 10\end{array}$

$\begin{array}{ll}3.2 \text { Zero-modes equation } \delta \mathcal{R}=0 & 13\end{array}$

4 Tensor zero-modes of five-branes 14

$\begin{array}{lll}4.1 & \text { Zero-modes in the RR sector } & 15\end{array}$

$\begin{array}{ll}4.2 \text { Normalizability } & 16\end{array}$

$\begin{array}{lll}4.3 & \text { Self-duality constraint } & 18\end{array}$

5 Effective theories of locally non-geometric five-branes 20

5.1 R-brane type 20

5.2 Localized KK-monopole type 23

6 Conclusion and discussions $\quad 24$

A Quick introduction to double field theory 26

$\begin{array}{ll}\text { A.1 NSNS sector } & 26\end{array}$

$\begin{array}{lll}\text { A.2 RR sector } & 27\end{array}$

$\begin{array}{lr}\text { B Detailed calculations } & 29\end{array}$

B.1 Calculations on the generalized Ricci tensor 29

B.2 Calculations on the RR zero-modes 30

B.3 Calculations on the normalizability 34

B.4 Calculations on the self-duality constraint 35

\section{Introduction}

U-duality is a key ingredient to understand whole structure of M-theory and string theories [1]. The duality exhibits its remarkable features in lower dimensions. For example, M-theory compactified on $T^{D}$ shows the U-duality group $E_{D(D)}$ in the $(11-D)$-dimensional space-time. BPS states in lower dimensions, which originate from the higher-dimensional branes that wrap $T^{D}$, are classified by the U-duality group $[2,3]$. In addition to the conventional branes such as the D-branes and the NS5-branes and so on, there appear yet unfamiliar objects in the U-duality multiplets, known as the exotic branes [2-6]. Detailed classifications of exotic branes are found, for example, in $[7,8]$. 
The exotic branes are characterized by their highly non-perturbative nature in string theory. For example, they have tensions proportional to $g_{s}^{-\alpha}$ with $\alpha \geq 2$ which contrast to those of the D-branes given by the order $g_{s}^{-1}$. Here $g_{s}$ is the string coupling constant. Among other things, exotic branes appearing in the T-duality orbit of five-branes in type II theories have been intensively studied [5, 6, 9-13]. There are five-branes denoted as $5_{2}^{k}[3]$ related by T-duality transformations. The "2" in the lowerscript represents that the tensions of the five-branes are proportional to $g_{s}^{-2}$ while $k=0,1,2,3,4$ stands for the number of the isometries in the transverse directions in ten dimensions. For example, $k=0$ and $k=1$ correspond to the NS5-brane and the Kaluza-Klein (KK)-monopole. The $k=2$ case is an example of the exotic brane conventionally refered as Q-brane [14]. The geometry of the $5_{2}^{2}$-brane is patched together not by the diffeomorphism or gauge transformations but by T-duality transformations. In this sense, the background of the $5_{2}^{2}$-brane is known as a globally non-geometric space or a T-fold [15]. Although it is a codimension two brane and its existence as a stand alone object suffers from a subtle issue, one can explicitly write down the $5_{2}^{2}$-brane solution in the ordinary supergravity $[5,6]$. On the other hand, the $k=3$ brane has less obvious geometric meaning. One can not perform a T-duality transformation in the $5_{2}^{2}$-brane since any further isometries are forbidden there. However, from the viewpoint of the U-duality multiplet in lower dimensions, the $5_{2}^{3}$-brane should be allowed in higher dimensions. Nevertheless, it is less obvious whether that is a solution to supergravity or not.

Double field theory (DFT) [16], based on the doubled formalism developed in [17-19], is an effective gravity theory that has manifest T-duality in string theory. Due to the $O(D, D)$ covariance, one can treat the T-duality orbit of the branes without difficulty in DFT. The action of DFT is given by a generalized Ricci scalar and it reduces to the action of supergravity with the imposition of the strong constraint. Indeed, supergravity solutions such as the F-strings, the waves, the NS5-branes, the KK-monopoles and even the globally non-geometric objects, are found to be solutions to DFT [20, 21]. This is conceivable since DFT is, in a sense, a reformulation of supergravity. However, the most remarkable difference between the ordinary supergravity theories and DFT is the isometries for T-duality transformations. Contrary to supergravities, DFT does not necessarily require isometries to perform the T-duality transformation. Therefore, one can perform the formal (non-isometric) T-duality transformation to obtain geometries that are not solutions to supergravity. The $5_{2}^{3}$-brane is one of such kind of solution in DFT $[22,23]$. Due to the absence of the isometries, the $5_{2}^{3}$-brane geometry contains the dual coordinate $\tilde{x}$-dependence. This $\tilde{x}$-dependence is no longer removed even by the T-duality transformation and it is an essential nature of the new geometry. This kind of brane is known as an R-brane or the locally non-geometric object [14, 24, 25]. The geometry of the R-brane necessarily depends on the winding coordinate $\tilde{x}$. Therefore it loses the ordinary geometric meaning based on the point particle. We can continue this procedure further. The compactification on $T^{4}$ allows us to find the space-filling $5_{2}^{4}$-brane with the non-trivial winding coordinate dependence.

The other place that appears the winding coordinate dependence of geometries is the worldsheet instanton effects. This is first proposed in [26] where the dual side of the 
localized (unsmeared) NS5-brane is analyzed. In [27], it is shown that the isometry in the H-monopole (the NS5-brane smeared along the transverse direction) is broken by the string worldsheet instanton effects. In the dual KK-monopole side, the worldsheet instanton effects induce the dual coordinate dependence in the Taub-NUT geometry [28, 29]. The modified geometry is known as the localized KK-monopole [21]. The same is true even for the globally non-geometric $5_{2}^{2}$-brane [30-32]. The geometry is modified and characterized by the winding coordinate. We call it the localized Q5-brane. They are also classified into the locally non-geometric objects. A remarkable fact is that the localized KK-monopole and the localized Q5-brane are shown to be solutions to DFT [23]. The geometry with the winding corrections are necessary to understand a stringy nature of space-time and it is also discussed in other places [33, 34].

The purpose of this paper is to study the worldvolume effective theories of the locally non-geometric five-branes. Among other things, we focus on the R5-brane ( $5_{2}^{3}$-brane), the space-filling $5_{2}^{4}$-brane, the localized KK-monopole, Q5-brane and also the localized R5-brane, space-filling branes. For the globally non-geometric objects, the world-volume effective actions of the Dirac-Born-Infeld (DBI) type are obtained through the duality covariantization or formal T-duality transformations of known branes [35-40]. We here derive the effective theories of the locally non-geometric five-branes by the direct calculations within the formalism of DFT. We will explicitly determine the precise bosonic zero-modes of the five-branes and show that they are Nambu-Goldstone modes associated with the broken gauge symmetries in DFT. These zero-modes appear in the world-volume effective theories of the five-branes. We will show that these zero-modes are organized into the bosonic parts of the six-dimensional $\mathcal{N}=(1,1)$ vector and the $\mathcal{N}=(2,0)$ tensor multiplets.

The organization of this paper is as follows. In the next section, we briefly introduce five-brane solutions in DFT. In section 3, we determine the translational zero-modes in the doubled space. In section 4 , we determine the tensor zero-modes associated with the spontaneous breaking of the RR gauge symmetries in DFT. We will show that these zero-modes are organized into the desired six-dimensional supermultiplets. In section 5 , we focus on the world-volume effective theories of the locally non-geometric five-branes. Section 6 is conclusion and discussions. Appendix A devoted to a quick introduction to DFT. Appendix B contains the detailed calculations on the zero-modes.

\section{Locally non-geometric branes in double field theory}

In this section, we give a brief introduction to locally non-geometric branes, especially focusing on five-branes in DFT [23]. We consider a T-duality orbit of five-branes in type II string theories and exhibit the locally non-geometric objects in DFT.

The dynamical fields in DFT of type II string theories are given by the generalized metric $\mathcal{H}_{M N}$, the generalized dilaton $d$ and the $O(D, D)$ spinor $\chi \cdot^{1}$ The DFT action is

\footnotetext{
${ }^{1}$ Unless otherwise stated, we consider $D=10$ in the following.
} 
given by [41-43]:

$$
S_{\mathrm{DFT}}(\mathbb{S}, d, \chi)=\int \mathrm{d}^{2 D} X\left(e^{-2 d} \mathcal{R}(\mathcal{H}, d)+\frac{1}{4}(\not \partial \chi)^{\dagger} \mathbb{S}(\not \partial \chi)\right) .
$$

The generalized Ricci scalar $\mathcal{R}$ is defined by

$$
\begin{aligned}
\mathcal{R}= & 4 \mathcal{H}^{M N} \partial_{M} \partial_{N} d-\partial_{M} \partial_{N} \mathcal{H}^{M N}-4 \mathcal{H}^{M N} \partial_{M} d \partial_{N} d+4 \partial_{M} \mathcal{H}^{M N} \partial_{N} d \\
& +\frac{1}{8} \mathcal{H}^{M N} \partial_{M} \mathcal{H}^{K L} \partial_{N} \mathcal{H}_{K L}-\frac{1}{2} \mathcal{H}^{M N} \partial_{M} \mathcal{H}^{K L} \partial_{K} \mathcal{H}_{N L},
\end{aligned}
$$

where the indices $M, N, \ldots$ run over the $2 D$ dimensions. The inverse of the generalized metric $\mathcal{H}^{M N}$ is introduced by $\mathcal{H}^{M P} \mathcal{H}_{P N}=\delta^{M}{ }_{N}$ with its defining $O(D, D)$ property $\mathcal{H}^{M N}=\eta^{M P} \eta^{N Q} \mathcal{H}_{P Q}$. Here $\eta^{M N}$ and its inverse $\eta_{M N}$ are $O(D, D)$ invariant metric given by

$$
\eta_{M N}=\left(\begin{array}{cc}
0 & \delta^{\mu}{ }_{\nu} \\
\delta_{\mu}{ }^{\nu} & 0
\end{array}\right), \quad \eta^{M N}=\left(\begin{array}{cc}
0 & \delta_{\mu}{ }^{\nu} \\
\delta^{\mu}{ }_{\nu} & 0
\end{array}\right)
$$

The coordinate of the $2 D$-dimensional doubled space is decomposed as $X^{M}=\left(\tilde{x}_{\mu}, x^{\mu}\right)$, $(\mu=0, \ldots, D-1)$ where $x^{\mu}$ are the Fourier conjugate of the KK modes (the geometric coordinates) while $\tilde{x}_{\mu}$ are the Fourier conjugate of the winding modes (the winding coordinates). The $O(D, D)$ spinor $\chi$ is assumed to satisfy the self-duality constraint $\not \partial \chi=-\mathcal{K} \not \partial \chi$. Here $\not \partial=\Gamma^{M} \partial_{M}$ and $\Gamma^{M}$ are the $2 D$-dimensional gamma matrices. The operator $\mathcal{K}=C^{-1} \mathbb{S}$ is defined by the charge conjugation matrix $C$ and the spinor representation of the generalized metric $\mathbb{S}=\mathbb{S}^{\dagger} \in \operatorname{Spin}^{-}(D, D)$. See appendix A and [43] for the precise definitions.

The action $(2.1)$ makes the $O(D, D)$ T-duality be manifest and it is invariant under the following gauge transformations:

$$
\begin{aligned}
\delta_{\xi} \mathcal{H}_{M N} & =\widehat{\mathcal{L}}_{\xi} \mathcal{H}_{M N}=\xi^{P} \partial_{P} \mathcal{H}^{M N}+\left(\partial^{M} \xi_{P}-\partial_{P} \xi^{M}\right) \mathcal{H}^{P N}+\left(\partial^{N} \xi_{P}-\partial_{P} \xi^{N}\right) \mathcal{H}^{M P} \\
\delta_{\xi} d & =\widehat{\mathcal{L}}_{\xi} d=\xi^{M} \partial_{M} d-\frac{1}{2} \partial_{M} \xi^{M} \\
\delta_{\xi} \chi & =\widehat{\mathcal{L}}_{\xi} \chi=\xi^{M} \partial_{M} \chi+\frac{1}{2} \partial_{N} \xi_{M} \Gamma^{N} \Gamma^{M} \chi \\
\delta_{\lambda} \chi & =\not \partial \lambda
\end{aligned}
$$

where $\widehat{\mathcal{L}}_{\xi}$ is the generalized Lie derivative and $\xi^{M}, \lambda$ are parameters of a doubled vector and an $O(D, D)$ spinor. The gauge invariance of the DFT action (2.1) is guaranteed by the so-called strong constraint $\partial^{M} * \partial_{M} *=0$. Here $*$ stands for any dynamical fields and the gauge parameters in DFT.

It is convenient to parametrize the DFT fields in terms of the ordinary supergravity fields:

$$
\mathcal{H}_{M N}=\left(\begin{array}{cc}
g^{\mu \nu} & -g^{\mu \rho} B_{\rho \nu} \\
B_{\mu \rho} g^{\rho \nu} & g_{\mu \nu}-B_{\mu \rho} g^{\rho \sigma} B_{\sigma \nu}
\end{array}\right), \quad e^{-2 d}=\sqrt{-g} e^{-2 \Phi},
$$


where $g_{\mu \nu}, B_{\mu \nu}, \Phi$ are identified with the space-time metric, the NSNS $B$-field and the dilaton in type II supergravities. Meanwhile, the $O(D, D)$ spinor is expanded as

$$
\chi=\sum_{p=0}^{D} \frac{1}{p !} C_{\mu_{1} \cdots \mu_{p}} \psi^{\mu_{1}} \cdots \psi^{\mu_{p}}|0\rangle,
$$

where $C_{\mu_{1} \cdots \mu_{p}}$ are identified with the RR $p$-forms. ${ }^{2}$

The equations of motion in DFT are given by

$$
\mathcal{R}_{M N}+e^{2 d} \mathcal{E}_{M N}=0, \quad \mathcal{R}=0, \quad \not \partial(\mathcal{K} \not \chi \chi)=0 .
$$

Here the generalized Ricci tensor $\mathcal{R}_{M N}$ and the energy-momentum tensor $\mathcal{E}_{M N}$ are defined by

$$
\mathcal{R}_{M N}=P_{M N}{ }^{K L} \mathcal{K}_{K L}, \quad \mathcal{E}^{M N}=-\frac{1}{16} \mathcal{H}^{(M} \overline{\not \partial \chi} \Gamma^{N) P} \not \partial \chi .
$$

The projection operator $P$ and the tensor $K$ are given by the following expressions:

$$
\begin{aligned}
P_{M N}{ }^{K L}= & \frac{1}{2}\left[\delta_{M}{ }^{(K} \delta_{N}{ }^{L)}-\mathcal{H}_{M P} \eta^{P(K} \eta_{N Q} \mathcal{H}^{L) Q}\right] \\
\mathcal{K}_{M N}= & \frac{1}{8} \partial_{M} \mathcal{H}^{K L} \partial_{N} \mathcal{H}_{K L}-\frac{1}{4}\left(\partial_{L}-2 \partial_{L} d\right) \mathcal{H}^{K L} \partial_{K} \mathcal{H}_{M N}+2 \partial_{M} \partial_{N} d \\
& -\frac{1}{2} \partial_{(M} \mathcal{H}^{K L} \partial_{L} \mathcal{H}_{N) K}+\frac{1}{2}\left(\partial_{L}-2 \partial_{L} d\right)\left[\mathcal{H}^{K L} \partial_{(M} \mathcal{H}_{N) K}+\eta^{K P} \eta^{L Q} \mathcal{H}_{P(M} \partial_{K} \mathcal{H}_{N) Q}\right] .
\end{aligned}
$$

We use the convention of the (anti)symmetrization with the weight factor such as $A_{(M} B_{N)}=\left(A_{M} B_{N}+A_{N} B_{M}\right) / 2$.

Now we introduce the five-brane solutions in DFT. We consider the ansatz of the localized DFT monopole [21]:

$$
H_{M N}=\left(\begin{array}{cccc}
\eta^{m n} & 0 & 0 & 0 \\
0 & H^{-1} \delta^{a b} & 0 & -H^{-1} b^{a}{ }_{b} \\
0 & 0 & \eta_{m n} & 0 \\
0 & H^{-1} b_{a}{ }^{b} & 0 & H \delta_{a b}
\end{array}\right), \quad d=\text { const. }-\frac{1}{2} \log H, \quad \chi=0
$$

We introduce a new decomposition of the doubled coordinate:

$$
X^{M}=\left(\bar{x}_{m}, \bar{y}_{a} ; x^{m}, y^{a}\right)
$$

where $x^{m}(m=0,1, \ldots, 5), y^{a}(a=6, \ldots, 9)$ are the worldvolume and the transverse coordinates and $\bar{x}_{m}, \bar{y}_{a}$ are their duals. We here stress that we never give the physical meaning of the coordinates $\left(x^{m}, y^{a}, \bar{x}_{m}, \bar{y}_{a}\right)$ at this stage. Namely, the roles of the geometric and the winding coordinates are fixed through the following $O(D, D)$ assignment:

$$
X^{M}=\Omega_{N}^{M} X^{N}, \quad \Omega \in O(D, D) .
$$

\footnotetext{
${ }^{2}$ We follow the convention of the RR $p$-forms employed in $[42,43]$.
} 
One should be careful about the distinction between $X^{M}=\left(\tilde{x}_{\mu}, x^{\mu}\right)$ and $X^{M}$ in (2.12). Likewise, the generalized metric (2.5) parametrized in the space-time metric $g$ and the NSNS $B$-field are read off by the ansatz (2.11) through the corresponding $O(D, D)$ assignment:

$$
\mathcal{H}_{M N}=H_{K L} \Omega^{K}{ }_{M} \Omega^{L}{ }_{N}, \quad \Omega \in O(D, D) .
$$

For the function $H$ appearing in the ansatz (2.11), we assume that it is a function of the transverse coordinate $y^{a}$. Together with a trivial condition $\bar{\partial}^{a} *=0$ to the strong constraint, one can show that the ansatz (2.11) satisfies the equations motion (2.7) when the following conditions are satisfied [23]:

$$
3 \partial_{[a} b_{b c]}=\varepsilon_{a b c d} \partial^{d} H(y), \quad \square H=0 .
$$

Here $b_{a b}$ is an antisymmetric tensor depending on $y^{a}, \varepsilon_{a b c d}$ is the Levi-Civita symbol, and $\square=\delta^{a b} \frac{\partial}{\partial y^{a}} \frac{\partial}{\partial y^{b}}$ is the flat Laplacian defined by $y^{a}$. Therefore $H$ is a harmonic function in the $y$-space. The equations (2.15) correspond to the BPS condition for the NS5-brane in ordinary supergravity. We stress that the condition (2.15) allows for five-branes of any codimensions, namely, $H$ is a harmonic function in 0 to 4 dimensions.

In the following, we will fix specific T-duality frames by giving the $O(D, D)$ matrices $\Omega$ explicitly and write down the five-brane solutions in each frame.

NS5-brane $\left(\mathbf{5}_{\mathbf{2}}^{\mathbf{0}}\right)$. When the $O(D, D)$ matrix is given by $\Omega=1$, the role of the geometric coordinates is assigned to $x^{\mu}=\left(x^{m}, y^{a}\right)$. The role of the winding coordinates is assigned to $\tilde{x}_{\mu}=\left(\bar{x}_{m}, \bar{y}_{a}\right)$. According to the relation (2.14), the metric, the $B$-field and the dilaton are read off

$$
\begin{aligned}
\mathrm{d} s^{2} & =\eta_{m n} \mathrm{~d} x^{m} \mathrm{~d} x^{n}+H \delta_{a b} \mathrm{~d} y^{a} \mathrm{~d} y^{b}, \\
B & =\frac{1}{2} b_{a b} \mathrm{~d} y^{a} \wedge \mathrm{d} y^{b}, \\
e^{2 \Phi} & =H .
\end{aligned}
$$

This is nothing but the NS5-brane solution in type II supergravities. It is obvious that the world-volume of the NS5-brane extends to the $x^{0}, \cdots, x^{5}$ directions. The harmonic function $H(r)$ is defined in the transverse directions $y^{6}, \cdots, y^{9}$ and is given by

$$
H(r)=c_{1}+\frac{c_{2}}{r^{2}}, \quad r^{2}=\delta_{a b} y^{a} y^{b} .
$$

Here $c_{1}, c_{2}$ are appropriate constants. Note that the explicit form of $b_{a b}$ is determined through the BPS condition (2.15).

KK-monopole $\left(\mathbf{5}_{\mathbf{2}}^{\mathbf{1}}\right)$. The KK-monopole is obtained by the T-duality transformation of the NS5-brane along a transverse direction. Remember that in order to perform the genuine T-duality transformations, we introduce isometries along the transverse directions. To do so, we smear the harmonic function along the $y^{9}$-direction in the NS5-brane. Then $H$ becomes

$$
H(r)=c_{1}+\frac{c_{2}}{r}, \quad r^{2}=\delta_{i j} y^{i} y^{j},
$$

where $c_{1}, c_{2}$ are again appropriate constants. 
We now give another $O(D, D)$ matrix $\Omega=h_{9}$, where $h_{\mathrm{k}}$ is a factorized T-duality transformation along the $y^{k}$-direction given by

$$
h_{\mathrm{k}}=\left(\begin{array}{cc}
1-e_{\mathrm{k}} & e_{\mathrm{k}} \\
e_{\mathrm{k}} & 1-e_{\mathrm{k}}
\end{array}\right), \quad\left(e_{\mathrm{k}}\right)_{\mu \nu}=\delta_{\mu \mathrm{k}} \delta_{\nu \mathrm{k}} .
$$

Then the assignment of the coordinates are $x^{\mu}=\left(x^{m}, y^{i}, \bar{y}_{9}\right),(i=6,7,8)$ and $\tilde{x}_{\mu}=$ $\left(\bar{x}_{m}, \bar{y}_{i}, y^{9}\right)$. The BPS equation (2.15) is solved by a Dirac monopole $B_{i 9}=A_{i}=b_{i 9}$ in the $\left(y^{6}, y^{7}, y^{8}\right)$-plane. The other components of the $B$-field are all zero. Then we write down the explicit solution as

$$
\begin{aligned}
\mathrm{d} s^{2} & =\eta_{m n} \mathrm{~d} x^{m} \mathrm{~d} x^{n}+H^{-1}\left(\mathrm{~d} \bar{y}_{9}+A_{i} \mathrm{~d} y^{i}\right)^{2}+H \delta_{i j} \mathrm{~d} y^{i} \mathrm{~d} y^{j}, \\
B & =0 \\
e^{2 \Phi} & =\text { const. }
\end{aligned}
$$

This is nothing but the KK-monopole of codimension three in type II supergravities. The transverse direction is characterized by the Taub-NUT space whose isometry direction is given by $\bar{y}_{9}$. The solution (2.20) is apparently geometric.

Q5-brane $\left(\mathbf{5}_{\mathbf{2}}^{\mathbf{2}}\right)$. The so-called exotic Q5-brane or $5_{2}^{2}$-brane is obtained by performing the T-duality transformation along the other direction in the KK-monopole. We now give yet another $O(D, D)$ matrix $\Omega=h_{8} \cdot h_{9}$. Then the geometric coordinates are $x^{\mu}=$ $\left(x^{m}, y^{\alpha}, \bar{y}_{8}, \bar{y}_{9}\right)(\alpha=6,7)$ while the winding ones are $\tilde{x}_{\mu}=\left(\bar{x}_{m}, \bar{y}_{\alpha}, y^{8}, y^{9}\right)$. The new isometry direction is given by $\bar{y}_{8}$. The harmonic function becomes

$$
H=c_{1}+c_{2} \log \frac{\mu}{r}, \quad r^{2}=\delta_{\alpha \beta} y^{\alpha} y^{\beta},
$$

where $c_{1}, c_{2}$ are constants and $\mu$ is a parameter that characterizes the effective description of defect branes as a stand alone object $[11,44]$. Then the resulting solution is given by

$$
\begin{aligned}
\mathrm{d} s^{2} & =\eta_{m n} \mathrm{~d} x^{m} \mathrm{~d} x^{n}+H \delta_{\alpha \beta} \mathrm{d} y^{\alpha} \mathrm{d} y^{\beta}+\frac{H}{H^{2}+A_{8}^{2}}\left[\mathrm{~d} \bar{y}_{9}^{2}+\mathrm{d} \bar{y}_{8}^{2}\right], \\
B & =-\frac{A_{8}}{H^{2}+A_{8}^{2}} \mathrm{~d} \bar{y}_{8} \wedge \mathrm{d} \bar{y}_{9}, \\
e^{2 \Phi} & =\frac{H}{H^{2}+A_{8}^{2}} .
\end{aligned}
$$

Here

$$
A_{8}=-c_{2} \arctan \left(\frac{y^{7}}{y^{6}}\right)
$$

is the smeared Dirac monopole in a specific gauge. The solution (2.22) has a non-trivial $O(2,2)$ monodromy around the brane core and the geometry is not patched together by the diffeomorphism or the $B$-field gauge transformation. Therefore the solution is not geometric in the usual sense and it is called a globally non-geometric background or a T-fold [15]. However, it is obvious that the solution (2.22) is written in the geometric coordinate and is indeed a solution to supergravity $[5,6]$. 
R5-brane $\left(\mathbf{5}_{2}^{\mathbf{3}}\right)$. We now try to make further T-duality transformation along another transverse direction. To this end, we give $\Omega=h_{7} \cdot h_{8} \cdot h_{9}$. Then $x^{\mu}=\left(x^{m}, y^{6}, \bar{y}_{\hat{m}}\right),(\hat{m}=$ $7,8,9)$ is the geometric and $\tilde{x}_{\mu}=\left(\bar{x}_{m}, \bar{y}_{6}, y^{\hat{m}}\right)$ are the winding coordinates. The harmonic function becomes

$$
H=c_{1}+c_{2}\left|y^{6}\right| .
$$

Here $c_{1}, c_{2}$ are constants again. Then the solution reads

$$
\begin{aligned}
\mathrm{d} s^{2} & =\eta_{m n} \mathrm{~d} x^{m} \mathrm{~d} x^{n}+H\left(\mathrm{~d} y^{6}\right)^{2}+H^{-1} \mathrm{~d} \bar{y}_{7}^{2}+\frac{H}{H^{2}+A_{8}^{2}}\left[\mathrm{~d} \bar{y}_{8}^{2}+\mathrm{d} \bar{y}_{9}^{2}\right], \\
B & =-\frac{A_{8}}{H^{2}+A_{8}^{2}} \mathrm{~d} \bar{y}_{8} \wedge \mathrm{d} \bar{y}_{9}, \\
e^{2 \Phi} & =\frac{1}{H^{2}+A_{8}^{2}},
\end{aligned}
$$

where the smeared Dirac monopole becomes

$$
A_{8}=c_{2}\left(\operatorname{sgn} y^{6}\right) y^{7} .
$$

Due to the BPS condition (2.15), the $B$-field should depend on $y^{7}$. A remarkable feature is that the solution depends on $y^{7}$ which is the winding coordinate. The solution (2.25) is known as the R5-brane [14]. Since it is impossible to represent the solution only by the geometric coordinates but it should include the dual winding coordinates $\tilde{x}_{7}=y^{7}$, this is called a locally non-geometric solution. We stress that this is not a solution to conventional supergravity anymore but must be in string theory. However, it is discussed that locally non-geometric branes of domain wall type are solutions to deformed supergravities [7].

SF5-brane $\left(\mathbf{5}_{\mathbf{2}}^{\mathbf{4}}\right)$. Finally, we consider a space-filling (SF) 5 -brane $[7,23]$. This is obtained by performing the T-duality transformation along the final transverse direction. Given $\Omega=h_{6} \cdot h_{7} \cdot h_{8} \cdot h_{9}$, the geometric coordinates are $x^{\mu}=\left(x^{m}, \bar{y}_{a}\right)(a=6,7,8,9)$ while the winding ones are $\tilde{x}_{\mu}=\left(\bar{x}_{m}, y^{a}\right)$. The solution becomes

$$
\begin{aligned}
\mathrm{d} s^{2} & =\eta_{m n} \mathrm{~d} x^{m} \mathrm{~d} x^{n}+H^{-1} \delta^{a b} \mathrm{~d} \bar{y}_{a} \mathrm{~d} \bar{y}_{b}-\frac{H^{-1}}{H^{2}+A_{8}^{2}}\left[A_{8}^{2} \mathrm{~d} \bar{y}_{8}^{2}+A_{8}^{2} \mathrm{~d} \bar{y}_{9}^{2}\right], \\
B & =-\frac{A_{8}}{H^{2}+A_{8}^{2}} \mathrm{~d} \bar{y}_{8} \wedge \mathrm{d} \bar{y}_{9}, \\
e^{2 \Phi} & =\frac{H^{-1}}{H^{2}+A_{8}^{2}} .
\end{aligned}
$$

Here the harmonic function and the gauge field are given by

$$
H=c_{1}+c_{2}\left|y^{6}\right|, \quad A_{8}=\frac{c_{2}}{2}\left(\operatorname{sgn} y^{6}\right) y^{7} .
$$

We stress that $y^{6}, y^{7}$ are the winding coordinates and the solution (2.27) loses the nature of the conventional geometry. This again shows the locally non-geometric property. We note that if we introduce the complete isometries along the four transverse directions by the smearing, the geometry of the space-filling brane becomes trivial, namely, $H$ and $A_{8}$ are constants and no non-trivial structure would be left. 


\section{Translational zero-modes in doubled space}

In this section, we determine the translational zero-modes of the five-branes in the doubled space. As for the ordinary branes in supergravity, the five-brane solutions discussed in the previous section break the translational symmetries along the transverse directions to the brane world-volume. We remark that for the five-branes in the previous section, the transverse directions are realized by the $2 \times 4=8$ dimensional doubled space. The broken symmetries of the solutions give rise to the Nambu-Goldstone modes in DFT. The translational symmetry in the doubled space is represented by the generalized Lie derivative which is a part of the gauge symmetry in (2.4). Once a solution $\left(\mathcal{H}_{M N}^{(0)}, d^{(0)}, \chi^{(0)}\right)$ to DFT is found, we consider a fluctuation of fields around the solution:

$$
\mathcal{H}_{M N}^{(0)} \rightarrow \mathcal{H}_{M N}^{(0)}+\delta \mathcal{H}_{M N}, \quad d^{(0)} \rightarrow d^{(0)}+\delta d, \quad \chi^{(0)} \rightarrow \chi^{(0)}+\delta \chi
$$

where the variation $\delta$ is generated by broken symmetries. We then substitute the fluctuations (3.1) into the equations of motion (2.7) and determine the zero-modes of the solution.

Given the fluctuations (3.1) around the five-brane solution (2.11), the variations of the equations of motion generated by the generalized Lie derivative $\widehat{\mathcal{L}}_{\xi}$ become

$$
\delta \mathcal{R}_{M N}=0, \quad \delta \mathcal{R}=0 .
$$

Note that since the $O(D, D)$ spinor $\chi$ all vanishes in the ansatz (2.11) and it never breaks the translational symmetry, the energy-momentum tensor $\mathcal{E}^{M N}$ does not contribute to the equations (3.2). In order to evaluate the variations in (3.2), we decompose the generalized Ricci scalar and tensor into the component form by using the following parametrization of the ansatz (2.11):

$$
\begin{aligned}
& H_{M N}=\left(\begin{array}{cc}
g^{\mu \nu} & -g^{\mu \rho} B_{\rho \nu} \\
B_{\mu \rho} g^{\rho \nu} & g_{\mu \nu}-B_{\mu \rho} g^{\rho \sigma} B_{\sigma \nu}
\end{array}\right), \quad g_{\mu \nu}=\left(\begin{array}{cc}
\eta_{m n} & 0 \\
0 & H(x) \delta_{a b}
\end{array}\right), \quad B_{\mu \nu}=\left(\begin{array}{ll}
0 & 0 \\
0 & b_{a b}(x)
\end{array}\right), \\
& d=\Phi-\frac{1}{4} \log |g|, \quad \Phi=\text { const. }+\frac{1}{2} \log H(x),
\end{aligned}
$$

where $g, B$ are $D \times D$ symmetric and skew-symmetric matrices and $\Phi$ is a scalar function. One should again keep in mind that their roles are not fixed at this stage. These matrices and scalar are identified with the space-time metric $g_{\mu \nu}$, the $B$-field $B_{\mu \nu}$ and the dilation $\Phi$ through the $O(D, D)$ assignment (2.13) and (2.14). It is worthwhile to note that, with the imposition of the strong constraint, the generalized Lie derivative $\widehat{\mathcal{L}}_{\xi}$ by $\xi^{M}=\left(\bar{\epsilon}_{\mu}, \epsilon^{\mu}\right)$ on the generalized metric and the dilaton (3.3) results in the linear combination of the ordinary Lie derivative $\mathcal{L}_{\epsilon}$ and the gauge transformation $\delta_{\bar{\epsilon}}$ in their components. Namely, we have

$$
\begin{gathered}
\delta H_{M N}=\widehat{\mathcal{L}}_{\xi} H_{M N} \stackrel{\bar{\partial}^{\mu} *=0}{\longrightarrow}\left\{\begin{array}{l}
\delta g_{\mu \nu}=\mathcal{L}_{\epsilon} g_{\mu \nu} \\
\delta B_{\mu \nu}=\mathcal{L}_{\epsilon} B_{\mu \nu}+2 \partial_{[\mu} \bar{\epsilon}_{\nu]}
\end{array}\right. \\
\delta d=\widehat{\mathcal{L}}_{\xi} d \stackrel{\bar{\partial}^{\mu} *=0}{\longrightarrow} \delta \Phi=\mathcal{L}_{\epsilon} \Phi .
\end{gathered}
$$


Before writing down the explicit variations of the components, we note the fact that the precise Nambu-Goldstone modes in a curved space are given by the constant moduli, which will be promoted to the world-volume fields, supplemented by appropriate weight factors [45]. Following the analysis in the DFT wave solution [20], we first try the ansatz for the gauge parameters $\xi^{M}=\left(\bar{\epsilon}_{\mu}, \epsilon^{\mu}\right)$ given by

$$
\epsilon^{a}=H^{s} \phi_{0}^{a}, \quad \bar{\epsilon}_{a}=H^{s} \bar{\phi}_{0 a}, \quad(a=6,7,8,9)
$$

where $\phi_{0}^{a}, \bar{\phi}_{0 a}$ are the constant moduli parameters, $H$ is the harmonic function in the solution (2.15) and $s$ is a constant. However, one easily finds that the naive ansatz (3.5) allows only constant $\phi_{0}^{a}, \bar{\phi}_{0 a}$ and it is not suitable for the world-volume fields. Instead, we mimic the ansatz discussed in the analysis on the M5-brane [45]:

$$
\epsilon^{a}=H^{s} \phi_{0}^{a}, \quad \bar{\epsilon}_{a}=-H^{s} \phi_{0}^{b} b_{b a} .
$$

The constant $s$ will be determined later. We will see that (3.6) is the correct ansatz in due course. Then the explicit variation of the relevant components are given by

$$
\begin{aligned}
\delta g_{a b} & =\mathcal{L}_{\epsilon} g_{a b}=H^{s}\left[2 s \phi_{0(a} \partial_{b)} H+\delta_{a b} \phi_{0}^{c} \partial_{c} H\right], \\
\delta B_{a b} & =\mathcal{L}_{\epsilon} B_{a b}+2 \partial_{[a} \bar{\epsilon}_{b]}=H^{s} \phi_{0}^{c} H_{a b c}=H^{s} \phi_{0}^{c} \varepsilon_{a b c d} \partial^{d} H, \\
\delta \Phi & =\mathcal{L}_{\epsilon} \Phi=H^{s} \phi_{0}^{a} \partial_{a} \Phi=\frac{1}{2} H^{s-1} \phi_{0}^{a} \partial_{a} H
\end{aligned}
$$

where $H_{\mu \nu \rho}=3 \partial_{[\mu} B_{\nu \rho]}$ and we have used the BPS condition (2.15) in the evaluation of $\delta B_{a b}$.

Finally, fields that characterize the world-volume effective theory of a brane are defined by promoting the constant moduli to functions on the world-volume:

$$
\phi_{0}^{a} \rightarrow \phi^{a}(x),
$$

where $x$ is the coordinate of the brane world-volume. We call these fields the fluctuation zero-modes of the five-branes. In the following, we evaluate the equations (3.2) for the variations (3.7) with the fields (3.8) and determine the kinematics that governs the fluctuation zero-modes.

\subsection{Zero-modes equation $\delta \mathcal{R}_{M N}=0$}

We first evaluate the variation of the generalized Ricci tensor. It is convenient to rewrite the components of the generalized Ricci tensor as

$$
\begin{aligned}
& \left.\mathcal{R}_{\mu \nu}=\frac{1}{2}(g \text { eq. })_{\mu \nu}-\left(B g^{-1}\right)_{\left(\left.\mu\right|^{\beta}\right.}(B \text { eq. })_{\mid \nu) \beta}-\frac{1}{2}\left(B g^{-1}\right)_{\mu}{ }^{\alpha}\left(B g^{-1}\right)_{\nu}{ }^{\beta} \text { (g eq. }\right)_{\alpha \beta}, \\
& \left.\mathcal{R}_{\mu}{ }^{\nu}=-\frac{1}{2} g^{\nu \beta}(B \text { eq. })_{\mu \beta}-\frac{1}{2}\left(B g^{-1}\right)_{\mu}{ }^{\alpha} g^{\nu \beta} \text { (g eq. }\right)_{\alpha \beta}, \\
& \mathcal{R}^{\mu \nu}=-\frac{1}{2} g^{\mu \alpha} g^{\nu \beta}(g \text { eq. })_{\alpha \beta},
\end{aligned}
$$


where we have defined the following expressions:

$$
\begin{aligned}
\text { (g eq. })_{\mu \nu} & =R_{\mu \nu}-\frac{1}{4} H_{\mu \rho \sigma} H_{\nu}^{\rho \sigma}+2 \nabla_{\mu} \nabla_{\nu} \Phi \\
(B \text { eq. })_{\mu \nu} & =\frac{1}{2} \nabla^{\alpha} H_{\alpha \mu \nu}-H_{\alpha \mu \nu} \nabla^{\alpha} \Phi \\
\left(B g^{-1}\right)_{\mu}{ }^{\nu} & =B_{\mu \rho} g^{\rho \nu}
\end{aligned}
$$

where $R_{\mu \nu}$ and $\nabla_{\mu}$ are the Ricci tensor and the covariant derivative defined by $g_{\mu \nu}$. The derivation of the expressions (3.9) is found in appendix B. Then, the variations of the generalized Ricci tensor become

$$
\begin{aligned}
\delta \mathcal{R}_{\mu \nu} & =\frac{1}{2} \delta(\text { g eq. })_{\mu \nu}-\left(B g^{-1}\right)_{\left(\left.\mu\right|^{\rho}\right.} \delta(B \text { eq. })_{\mid \nu) \rho}-\frac{1}{2}\left(B g^{-1}\right)_{\mu}{ }^{\rho}\left(B g^{-1}\right)_{\nu}{ }^{\sigma} \delta(g \text { eq. })_{\rho \sigma}, \\
\delta \mathcal{R}_{\mu}{ }^{\nu} & \left.=-\frac{1}{2} g^{\nu \rho} \delta \text { (B eq. }\right)_{\mu \rho}-\frac{1}{2} g^{\nu \rho}\left(B g^{-1}\right)_{\mu}{ }^{\sigma} \delta(g \text { eq. })_{\rho \sigma} \\
\delta \mathcal{R}^{\mu \nu} & \left.=-\frac{1}{2} g^{\mu \rho} g^{\nu \sigma} \delta \text { (g eq. }\right)_{\rho \sigma} .
\end{aligned}
$$

Therefore, we find that the equations for the zero-modes are given by

$$
\delta(\text { g eq. })_{\mu \nu}=0, \quad \delta(B \text { eq. })_{\mu \nu}=0 .
$$

Variation $\delta$ (g eq.). We first analyze the variation $\delta(g \text { eq. })_{\mu \nu}$. This is explicitly given by

$$
\delta(\text { g eq. })_{\mu \nu}=\delta R_{\mu \nu}-\frac{1}{4} \delta\left(H_{\mu \rho \sigma} H_{\nu}^{\rho \sigma}\right)+2 \delta\left(\nabla_{\mu} \nabla_{\nu} \Phi\right) .
$$

In the following, we write down the equation (3.13) by decomposing the indices into the world-volume and the transverse directions. Using the variations (3.7), the non-zero variations of the Christoffel symbol defined by $g_{\mu \nu}$ for the localized DFT monopole (2.11) are found to be

$$
\begin{aligned}
\delta \Gamma_{a b}^{m}=- & \frac{1}{2} H^{s}\left[2 s\left(\partial^{m} \phi_{(a}\right) \partial_{b)} H+\delta_{a b}\left(\partial^{m} \phi^{d}\right) \partial_{d} H\right], \\
\delta \Gamma_{m b}^{c}=\frac{1}{2} & H^{s-1}\left[s\left(\left(\partial_{m} \phi^{c}\right) \partial_{b} H+\left(\partial_{m} \phi_{b}\right) \partial^{c} H\right)+\delta_{b}^{c}\left(\partial_{m} \phi^{d}\right) \partial_{d} H\right], \\
\delta \Gamma_{a b}^{c}=\frac{1}{2}[ & 2(s-1) H^{s-2}\left(s \phi^{c} \partial_{a} H \partial_{b} H+\delta_{(a \mid}^{c} \phi^{d} \partial_{d} H \partial_{\mid b)} H\right) \\
& \quad+s H^{s-2}\left(\delta_{a b} \phi^{c}(\partial H)^{2}-2 \phi_{(a} \partial_{b)} H \partial^{c} H\right) \\
& \left.\quad+H^{s-2} \delta_{a b} \phi^{d} \partial_{d} H \partial^{c} H+H^{s-1}\left(2 s \phi^{c} \partial_{a} \partial_{b} H+2 \delta_{(a \mid}^{c} \phi^{d} \partial_{d} \partial_{\mid b)} H-\delta_{a b} \phi^{d} \partial_{d} \partial^{c} H\right)\right],
\end{aligned}
$$

where $m, n, \ldots=0,1, \ldots, 5$ and $a, b, \ldots=6, \ldots, 9$ are the world-volume and the transverse directions, respectively. Then, we find the variations of the Ricci tensor are given by

$$
\begin{aligned}
\delta R_{m n}= & -(s+2) H^{s-1}\left(\partial_{m} \partial_{n} \phi^{c}\right) \partial_{c} H \\
\delta R_{m b}= & -\frac{1}{2} H^{s-2}\left[\left(s^{2}+s-3\right)\left(\partial_{m} \phi^{c}\right) \partial_{c} H \partial_{b} H-s(s+1)\left(\partial_{m} \phi_{b}\right)(\partial H)^{2}\right] \\
& -\frac{1}{2} H^{s-1}\left[(s+3)\left(\partial_{m} \phi^{c}\right) \partial_{c} \partial_{b} H-s\left(\partial_{m} \phi_{b}\right) \square H\right]
\end{aligned}
$$




$$
\begin{aligned}
\delta R_{a b}= & -\frac{1}{2} H^{s}\left[2 s\left(\partial_{m} \partial^{m} \phi_{(a}\right) \partial_{b)} H+\delta_{a b}\left(\partial_{m} \partial^{m} \phi^{c}\right) \partial_{c} H\right] \\
& +\frac{1}{2}\left[H^{s-2} \delta_{a b} \phi^{c} \partial_{c} H \square H-2 s H^{s-2} \phi_{(a} \partial_{b)} H \square H-H^{s-1} \delta_{a b} \phi^{c} \partial_{c} \square H\right] \\
& +3(s-1) H^{s-3} \phi^{c} \partial_{c} H \partial_{a} H \partial_{b} H-H^{s-1} \phi^{c} \partial_{c} \partial_{a} \partial_{b} H \\
& +H^{s-2}\left[\phi^{c} \partial_{c} H \partial_{a} \partial_{b} H-(2 s-3) \phi^{c} \partial_{c} \partial_{(a} H \partial_{b)} H\right]
\end{aligned}
$$

Here we have defined $(\partial H)^{2}=\delta^{a b} \partial_{a} H \partial_{b} H$. We next evaluate the variation

$$
\begin{aligned}
\delta\left(H_{3}^{2}\right)_{\mu \nu} & =\delta\left(H_{\mu \rho \sigma} H_{\nu}^{\rho \sigma}\right) \\
& =g^{\rho \tau} g^{\sigma \kappa}\left(\delta H_{\mu \rho \sigma}\right) H_{\nu \tau \kappa}+g^{\rho \tau} g^{\sigma \kappa} H_{\mu \rho \sigma}\left(\delta H_{\nu \tau \kappa}\right)+2\left(\delta g^{\rho \tau}\right) g^{\sigma \kappa} H_{\mu \rho \sigma} H_{\nu \tau \kappa} .
\end{aligned}
$$

Since we have only the following non-zero components for the localized DFT monopole,

$$
\begin{aligned}
\delta H_{m a b}= & H^{s}\left(\partial_{m} \phi^{c}\right) \varepsilon_{a b c d} \partial^{d} H \\
\delta H_{a b c}= & s H^{s-1} \phi^{d}\left(\varepsilon_{a b d e} \partial_{c} H+\varepsilon_{b c d e} \partial_{a} H+\varepsilon_{c a d e} \partial_{b} H\right) \partial^{e} H \\
& +H^{s} \phi^{d}\left(\varepsilon_{a b d e} \partial^{e} \partial_{c} H+\varepsilon_{b c d e} \partial^{e} \partial_{a} H+\varepsilon_{c a d e} \partial^{e} \partial_{b} H\right),
\end{aligned}
$$

the non-zero components of the variations are found to be

$$
\begin{aligned}
\delta\left(H_{3}^{2}\right)_{m a}= & 2 H^{s-2}\left(\left(\partial_{m} \phi_{a}\right)(\partial H)^{2}-\left(\partial_{m} \phi^{c}\right) \partial_{c} H \partial_{a} H\right), \\
\delta\left(H_{3}^{2}\right)_{a b}= & 4 H^{s-3}\left(s \phi_{(a} \partial_{b)} H(\partial H)^{2}-\delta_{a b} \phi^{c} \partial_{c} H(\partial H)^{2}-(s-1) \phi^{c} \partial_{c} H \partial_{a} H \partial_{b} H\right) \\
& +4 H^{s-2}\left(\delta_{a b} \phi^{c} \partial_{c} \partial_{d} H \partial^{d} H-\phi^{c} \partial_{c} \partial_{(a} H \partial_{b)} H+\phi_{(a} \partial_{b)} H \square H-\delta_{a b} \phi^{c} \partial_{c} H \square H\right) .
\end{aligned}
$$

Finally, we evaluate the variation

$$
\delta\left(\nabla_{\mu} \nabla_{\nu} \Phi\right)=\partial_{\mu} \partial_{\nu}(\delta \Phi)-\left(\delta \Gamma_{\mu \nu}^{\rho}\right) \partial_{\rho} \Phi-\Gamma_{\mu \nu}^{\rho} \partial_{\rho}(\delta \Phi) .
$$

By using (3.7) and (3.14), we find the non-zero components of the above variation are

$$
\begin{aligned}
\delta\left(\nabla_{m} \nabla_{n} \Phi\right)= & \frac{1}{2} H^{s-1}\left(\partial_{m} \partial_{n} \phi^{c}\right) \partial_{c} H, \\
\delta\left(\nabla_{m} \nabla_{a} \Phi\right)= & \frac{1}{4}\left[H^{s-2}\left((s-3)\left(\partial_{m} \phi^{c}\right) \partial_{c} H \partial_{a} H-s\left(\partial_{m} \phi_{a}\right)(\partial H)^{2}\right)+2 H^{s-1}\left(\partial_{m} \phi^{c}\right) \partial_{c} \partial_{a} H\right], \\
\delta\left(\nabla_{a} \nabla_{b} \Phi\right)=\frac{1}{2}\left(H^{s-2} \delta_{a b} \phi^{c} \partial_{c} \partial_{d} H \partial^{d} H+2(s-2) H^{s-2} \phi^{c} \partial_{c} \partial_{(a} H \partial_{b} H-H^{s-2} \phi^{c} \partial_{c} H \partial_{a} \partial_{b} H\right. & \quad-4(s-1) H^{s-3} \phi^{c} \partial_{c} H \partial_{a} H \partial_{b} H-H^{s-3} \delta_{a b} \phi^{c} \partial_{c} H(\partial H)^{2} \\
& \left.\quad+s H^{s-3} \phi_{(a} \partial_{b)} H(\partial H)^{2}+H^{s-1} \phi^{c} \partial_{c} \partial_{a} \partial_{b} H\right) .
\end{aligned}
$$

Collecting all together, we find that the equation of the zero-modes $\delta(g \text { eq. })_{\mu \nu}=0$ results in the following form:

$$
0=\delta(\text { g eq. })_{m n}=-(s+1) H^{s-1}\left(\partial_{m} \partial_{n} \phi^{c}\right) \partial_{c} H,
$$




$$
\begin{aligned}
0=\delta(\text { g eq. })_{m a}= & \frac{1}{2}(s+1)(s-1) H^{s-2}\left(\left(\partial_{m} \phi_{a}\right)(\partial H)^{2}-\left(\partial_{m} \phi^{c}\right) \partial_{c} H \partial_{a} H\right) \\
& -\frac{1}{2} H^{s-1}\left[(s+1)\left(\partial_{m} \phi^{c}\right) \partial_{c} \partial_{a} H-s\left(\partial_{m} \phi_{a}\right) \square H\right], \\
0=\delta(g \text { eq. })_{a b}= & -\frac{1}{2} H^{s}\left[2 s\left(\partial_{m} \partial^{m} \phi_{(a}\right) \partial_{b)} H+\delta_{a b}\left(\partial_{m} \partial^{m} \phi^{c}\right) \partial_{c} H\right] \\
& +\frac{1}{2}\left[3 H^{s-2} \delta_{a b} \phi^{c} \partial_{c} H \square H-2(s+1) H^{s-2} \phi_{(a} \partial_{b)} H \square H-H^{s-1} \delta_{a b} \phi^{c} \partial_{c} \square H\right] .
\end{aligned}
$$

Since $H$ is a harmonic function $\square H=0$, we find $s=-1$ and the scalar fields $\phi^{a}$ satisfy the Klein-Gordon equation $\partial_{m} \partial^{m} \phi^{a}=0$ in the six-dimensional world-volume.

Variation $\delta$ (B eq.). We next evaluate the variation

$$
\delta(B \text { eq. })_{\mu \nu}=\frac{1}{2} \delta\left(\nabla^{\alpha} H_{\alpha \mu \nu}\right)-\delta\left(H_{\alpha \mu \nu} \nabla^{\alpha} \Phi\right)
$$

One easily finds that $\delta(B \text { eq. })_{m n}=\delta(B \text { eq. })_{m a}=0$ for the localized DFT monopole. Therefore only non-zero component in this variation is

$$
\begin{aligned}
\delta(B \text { eq. })_{a b}= & \frac{1}{2} H^{s} \varepsilon_{a b c d}\left(\partial_{m} \partial^{m} \phi^{c}\right) \partial^{d} H+\frac{1}{2} H^{s-1} \phi^{c} \varepsilon_{a b c d} \partial^{d} \square H \\
& +(s-2) H^{s-2} \phi^{c}\left(\varepsilon_{a b[c \mid e} \partial^{e} \partial_{\mid d]} H+\varepsilon_{[a \mid c d e} \partial^{e} \partial_{\mid b]} H\right) \partial^{d} H .
\end{aligned}
$$

By using the condition $\square H=0$ and the relation

$$
\varepsilon_{[a b c \mid e} \partial^{e} \partial_{\mid d]} H=\frac{1}{2}\left(\varepsilon_{[a \mid c d e} \partial^{e} \partial_{\mid b]} H+\varepsilon_{a b[c \mid e} \partial^{e} \partial_{\mid d]} H\right)=0,
$$

which originates from the Bianchi identity $\partial_{[a} H_{b c d]}=0$, we have the zero-modes equation,

$$
0=\delta(B \text { eq. })_{a b}=\frac{1}{2} H^{s} \varepsilon_{a b c d}\left(\partial_{m} \partial^{m} \phi^{c}\right) \partial^{d} H .
$$

This is again satisfied by the Klein-Gordon scalar fields $\phi^{a}$.

\subsection{Zero-modes equation $\delta \mathcal{R}=0$}

We next evaluate the variation $\delta \mathcal{R}=0$. The generalized Ricci scalar in the parametrization (3.3) is given by

$$
\begin{aligned}
\mathcal{R}= & \frac{1}{4} g^{\mu \nu} \partial_{\mu} g^{\rho \sigma} \partial_{\nu} g_{\rho \sigma}-\frac{1}{2} g^{\mu \nu} \partial_{\nu} g^{\rho \sigma} \partial_{\sigma} g_{\mu \rho}-\frac{1}{12} H_{\mu \nu \rho} H^{\mu \nu \rho} \\
& +4 g^{\mu \nu} \partial_{\mu} \partial_{\nu} d-\partial_{\mu} \partial_{\nu} g^{\mu \nu}-4 g^{\mu \nu} \partial_{\mu} d \partial_{\nu} d+4 \partial_{\mu} g^{\mu \nu} \partial_{\nu} d
\end{aligned}
$$

It is convenient to rewrite the generalized Ricci scalar in terms of the well-known geometric quantities. A tedious rearrangement leads to the following expression

$$
\mathcal{R}=R+4\left(\nabla^{\mu} \nabla_{\mu} \Phi-(\nabla \Phi)^{2}\right)-\frac{1}{12} H_{\mu \nu \rho} H^{\mu \nu \rho}
$$


where we have used the relation $\partial_{\mu} d=\partial_{\mu} \Phi-\frac{1}{2} \Gamma_{\mu \rho}^{\rho}$ and $R$ is the ordinary Ricci scalar defined by

$$
R=g^{\mu \nu} R_{\mu \nu}=g^{\mu \nu}\left(\partial_{\rho} \Gamma_{\mu \nu}^{\rho}-\Gamma_{\rho \mu}^{\sigma} \Gamma_{\nu \sigma}^{\rho}-\partial_{\mu} \Gamma_{\nu \rho}^{\rho}+\Gamma_{\rho \sigma}^{\sigma} \Gamma_{\mu \nu}^{\rho}\right)
$$

Having an insight that (3.27) is the same form with the equation of motion for the dilaton $\Phi$ in the NSNS sector in supergravity, it is further convenient to rewrite the generalized Ricci scalar as

$$
\mathcal{R}=g^{\mu \nu}(\text { g eq. })_{\mu \nu}+2 \nabla^{\mu} \nabla_{\mu} \Phi-4(\nabla \Phi)^{2}+\frac{1}{6} H_{\mu \nu \rho} H^{\mu \nu \rho} .
$$

Then, the variation of the generalized Ricci scalar is given by

$$
\begin{aligned}
\delta \mathcal{R}= & \left.\left(\delta g^{\mu \nu}\right)(\text { g eq. })_{\mu \nu}+g^{\mu \nu} \delta \text { (g eq. }\right)_{\mu \nu} \\
& +2 \delta\left(g^{\mu \nu} \nabla_{\mu} \nabla_{\nu} \Phi\right)-4 \delta\left(g^{\mu \nu} \nabla_{\mu} \Phi \nabla_{\nu} \Phi\right)+\frac{1}{6} \delta\left(g^{\mu \nu} g^{\rho \sigma} g^{\tau \kappa} H_{\mu \rho \tau} H_{\nu \sigma \kappa}\right)
\end{aligned}
$$

With the help of the results in the previous paragraph, each term is evaluated as

$$
\begin{aligned}
\left(\delta g^{\mu \nu}\right)(\text { g eq. })_{\mu \nu} & =0 \\
g^{\mu \nu} \delta(\text { g eq. })_{\mu \nu} & =-(2 s+3) H^{s-1}\left(\partial_{m} \partial^{m} \phi^{c}\right) \partial_{c} H \\
2 \delta\left(g^{\mu \nu} \nabla_{\mu} \nabla_{\nu} \Phi\right) & =H^{s-1}\left(\partial_{m} \partial^{m} \phi^{c}\right) \partial_{c} H \\
-4 \delta\left(g^{\mu \nu} \nabla_{\mu} \Phi \nabla_{\nu} \Phi\right) & =3 H^{s-4} \phi^{c} \partial_{c} H(\partial H)^{2}-2 H^{s-3} \phi^{c} \partial_{c} \partial_{a} H \partial^{a} H, \\
\frac{1}{6} \delta\left(g^{\mu \nu} g^{\rho \sigma} g^{\tau \kappa} H_{\mu \rho \tau} H_{\nu \sigma \kappa}\right) & =-3 H^{s-4} \phi^{c} \partial_{c} H(\partial H)^{2}+2 H^{s-3} \phi^{c} \partial_{c} \partial_{a} H \partial^{a} H .
\end{aligned}
$$

Collecting all together, we obtain the equation for the translational zero-modes,

$$
0=\delta \mathcal{R}=-2(s+1) H^{s-1}\left(\partial_{m} \partial^{m} \phi^{c}\right) \partial_{c} H
$$

From this expression, we again find that $\phi^{a}$ are scalar fields satisfying the Klein-Gordon equation in the world-volume and $s=-1$. This is consistent with the results obtained in the analysis of $\delta \mathcal{R}_{M N}=0$.

In summary, we have the translational zero-modes given by

$$
\epsilon^{a}=H^{-1} \phi^{a}(x), \quad \bar{\epsilon}_{a}=-H^{-1} \phi^{b}(x) b_{b a},
$$

where the scalar fields $\phi^{a}$ satisfies the Klein-Gordon equation in the six-dimensional brane world-volume. These zero-modes are Nambu-Goldstone modes associated with the spontaneous breaking of the translational symmetry along the transverse doubled space to five-branes.

\section{Tensor zero-modes of five-branes}

In the previous section, we determined the zero-modes associated with the spontaneous breaking of the translational symmetry in the doubled space. In this section, we determine the tensor zero-modes associated with the spontaneous breaking of the gauge symmetry in the $\mathrm{RR}$ sector. 


\subsection{Zero-modes in the RR sector}

The localized DFT monopole (2.11) breaks the gauge symmetry of the RR potential in (2.4):

$$
\delta_{\lambda} \chi=\not \partial \lambda
$$

where $\lambda$ is an $O(D, D)$ spinor parameter. We examine a fluctuation $\delta_{\lambda} \chi$ which is given by the gauge transformation (4.1) in the localized DFT monopole solution. In order that, we employ the ansatz of the Nambu-Goldstone modes

$$
\lambda=e^{\frac{1}{2} b_{a b} \psi^{a} \psi^{b}} H^{t}(y) \bar{\lambda},
$$

where the $p$-form parameters in the constant $O(D, D)$ spinor $\bar{\lambda}$ extend along the worldvolume directions. The overall exponential factor guarantees that $H^{t}(y) \bar{\lambda}$ is the genuine zero-mode which is invariant under the NSNS $B$-field gauge transformation [43]. Here $H(y)$ is the harmonic function defined in the localized DFT monopole solution and $t$ is a real number which will be determined later.

Since the localized DFT monopole solution (2.11) is given within the solution to the strong constraint, we assume $\bar{\partial}^{\mu}=0$ in the following. Then the fluctuation (4.2) becomes

$$
\delta_{\lambda} \chi=e^{\frac{1}{2} b \psi \psi}\left[\frac{1}{2} H^{t} \partial_{c} b_{a b} \psi^{a b}+t H^{t-1} \partial_{c} H\right] \psi^{c} \bar{\lambda} .
$$

Here we have used the fact that $b$ has only the transverse components in the solution. We have also introduced the notation $b \psi \psi=b_{a b} \psi^{a} \psi^{b}$. In the following, we also use the notation such as $\psi^{\mu \nu \rho \cdots}=\psi^{\mu} \psi^{\nu} \psi^{\rho} \ldots$.

We next promote the constant parameter to the field in the world-volume:

$$
\bar{\lambda} \rightarrow \lambda(x) .
$$

The component expansion of the $O(D, D)$ spinor is given by

$$
\lambda(x)=\sum_{p=1}^{D} \frac{1}{(p-1) !} \lambda_{m_{1} \cdots m_{p-1}}(x) \psi^{m_{1} \cdots m_{p-1}}|0\rangle .
$$

Now we substitute the fluctuation into the equation of motion. The only relevant equation of motion for the RR zero-modes is

$$
\not \partial\left(\mathcal{K} \not \partial \delta_{\lambda} \chi\right)=0 .
$$

Here $\mathcal{K}=C^{-1} \mathbb{S}_{H}$. Substituting the fluctuation into the equation of motion and performing tedious calculations, we find (detailed calculations are found in appendix B),

$$
\begin{aligned}
& {\left[\left(t^{2}-1\right) H^{t-1} \partial_{a} H \partial_{a} H\right] \sum_{p} F^{l n_{1} \cdots n_{p-1}} \psi^{l n_{1} \cdots n_{p-1}}|0\rangle} \\
& -\left[\frac{1}{2} H^{t-1} \partial_{c} b_{e f} \psi^{e f c}+t H^{t} \partial_{c} H \psi^{c}\right] \sum_{p} p \partial_{m} F^{m n_{1} \cdots n_{p-1}} \psi^{n_{1} \cdots n_{p-1}}|0\rangle=0,
\end{aligned}
$$


where we have defined

$$
F^{m_{1} m_{2} \cdots m_{p}}=p ! \partial^{\left[m_{1}\right.} \lambda^{\left.m_{2} \cdots m_{p}\right]} .
$$

Therefore, we conclude that the zero-mode equation is satisfied when $t= \pm 1$ and

$$
\partial_{m} F^{m n_{1} \cdots n_{p-1}}=0 .
$$

The condition (4.9) is nothing but the Maxwell equation of the $p$-forms $\lambda$ in the sixdimensional world-volume.

\subsection{Normalizability}

In order to determine the value of $t$, we next examine the normalizability of the RR zeromodes. To this end, we evaluate the effective action of the $\mathrm{RR}$ zero-modes. This is obtained in the RR sector of the DFT action (2.1). We introduce the fluctuation $\delta_{\lambda} \chi$ given by the gauge transformation (4.3), with the promoted gauge parameter (4.4) in the action. Then the relevant term in the action becomes

$$
S_{\mathrm{DFT}, \mathrm{RR}}=\frac{1}{4} \int \mathrm{d}^{D} \tilde{x} \int \mathrm{d}^{D} x\left(\not \partial \delta_{\lambda} \chi\right)^{\dagger} \mathbb{S}_{H} \not \partial \delta_{\lambda} \chi,
$$

where $\mathbb{S}_{H}$ is the $O(D, D)$ spinor representation of the generalized metric for the localized DFT monopole solution (2.11). Once again, calculations give the following result (details are found in appendix B),

$$
\begin{aligned}
& \left(\not \partial \delta_{\lambda} \chi\right)^{\dagger} \mathbb{S}_{H}\left(\not \partial \delta_{\lambda} \chi\right)=-\frac{1}{4} H^{2 t-1} \partial_{c} b_{e f} \partial_{c^{\prime}} b_{e^{\prime} f^{\prime}} \sum_{p} f_{n m_{1} \cdots m_{p-1}} \\
& \times \sum_{q} f_{n^{\prime} m_{1}^{\prime} \cdots m_{q-1}^{\prime}} \eta^{n^{\prime} l^{\prime}} \eta^{m_{1}^{\prime} n_{1}^{\prime} \cdots \eta^{m_{q-1}^{\prime} n_{q-1}^{\prime}}} \\
& \times\left\langle 0\left|\psi_{m_{p-1} \cdots m_{1}} \psi_{c f e} \psi^{e^{\prime} f^{\prime} c^{\prime}} \psi^{l^{\prime} n_{1}^{\prime} \cdots n_{q-1}^{\prime}}\right| 0\right\rangle \\
& -t^{2} H^{2 t-1} \partial_{c} H \partial_{c^{\prime}} H \sum_{p} f_{n m_{1} \cdots m_{p-1}} \sum_{q} f_{n^{\prime} m_{1}^{\prime} \cdots m_{q-1}^{\prime}} \eta^{n^{\prime} l^{\prime}} \eta^{m_{1}^{\prime} n_{1}^{\prime}} \cdots \eta^{m_{q-1}^{\prime} n_{q-1}^{\prime}} \\
& \times\left\langle 0\left|\psi_{m_{p-1} \cdots m_{1}} \psi_{c} \psi^{c^{\prime}} \psi^{l^{\prime} n_{1}^{\prime} \cdots n_{q-1}^{\prime}}\right| 0\right\rangle .
\end{aligned}
$$

The doubled volume factor is decomposed into the world-volume and the transverse directions $\int \mathrm{d}^{D} \tilde{x} \mathrm{~d}^{D} x=\int_{\mathrm{wv} .} \mathrm{d}^{6} \tilde{x} \mathrm{~d}^{6} x \int_{\text {trans. }} \mathrm{d}^{4} \tilde{x} \mathrm{~d}^{4} x$. Here "wv." and "trans." mean the world-volume and the transverse spaces, respectively. The volume factors $\int_{\mathrm{wv} .} \mathrm{d}^{6} \tilde{x}$ and $\int_{\text {trans. }} \mathrm{d}^{4} \tilde{x}$ give finite values for the compactified torus. In order to obtain the world-volume theory, we now integrate out the transverse directions that survives the strong constraint:

$$
\int_{\text {trans. }} \mathrm{d}^{4} x\left(\not \partial \delta_{\lambda} \chi\right)^{\dagger} \mathbb{S}_{H}\left(\not \partial \delta_{\lambda} \chi\right)
$$

We evaluate the integrals of the first and the second terms in (4.11). The first term gives

$$
\begin{aligned}
& \int_{0}^{\infty} r^{3} \mathrm{~d} r H^{2 t-1} \partial_{[a} b_{b c]} \partial_{[d} b_{e f]}\left\langle 0\left|\psi_{a b c} \psi^{d e f}\right| 0\right\rangle \\
\sim & \int_{0}^{\infty} r^{3} \mathrm{~d} r H^{2 t-1} \partial_{g} H \partial_{h} H \varepsilon_{a b c g} \varepsilon_{d e f h} \delta_{a}{ }^{[d} \delta_{b}{ }^{e} \delta_{c}{ }^{f]} \\
\sim & \int_{0}^{\infty} r^{3} \mathrm{~d} r H^{2 t-1} \partial_{a} H \partial_{a} H .
\end{aligned}
$$


Here we have omitted the irrelevant overall factors. For the localized DFT monopole of the codimension four, we find $H=c_{1}+c_{2} r^{-2}$ and the relevant integral is given by

$$
\int_{0}^{\infty} \mathrm{d} r r^{-3}\left(c_{1}+c_{2} r^{-2}\right)^{2 t-1} .
$$

This is finite only when $t=-1$. For $t=1$, the integral diverges at $r=0$. Similarly, the second term in (4.11) also gives the integral (4.14), giving a finite result only when $t=-1$.

For the DFT monopole of the codimension three, we have $H=c_{1}+c_{2} r^{-1}$ and the relevant integral becomes

$$
\int_{0}^{\infty} \mathrm{d} r r^{-2}\left(c_{1}+c_{2} r^{-1}\right)^{2 t-1} .
$$

This again gives a finite result only when $t=-1$. For the five-brane of codimension two, we have

$$
H=c_{1}-c_{2} \ln r,
$$

where we have assumed $c_{1}, c_{2}>0$. The integral becomes

$$
I_{t}=\int_{0}^{\infty} \mathrm{d} r r^{-1}\left(c_{1}-c_{2} \ln r\right)^{2 t-1} .
$$

This diverges both for $t= \pm 1$. However, this is an artificial phenomenon originating from the fact that the codimension two brane is ill-defined as a stand alone object. Indeed, if we introduce a cutoff at $r=M$, this gives a finite result only when $t=-1$ :

$$
I_{t=-1}=\frac{1}{2 c_{2}\left(c_{1}-c_{2} \ln M\right)^{2}} .
$$

For the DFT monopole of the codimension one, we have,

$$
H=c_{1}+c_{2}|x|,
$$

where we have assumed that $c_{1}, c_{2}>0$. The relevant integral becomes

$$
\int_{-\infty}^{\infty} \mathrm{d} x\left(c_{1}+c_{2}|x|\right)^{2 t-1} .
$$

This is finite only when $t=-1$. Therefore, we conclude that $t=-1$ is the correct value of the normalizable zero-modes.

The same analysis is also applied in the NSNS sector. By using the explicit variations of the fields

$$
\delta g_{a b}=H^{-1}\left(\delta_{a b} \phi^{c} \partial_{c} H-2 \phi_{(a} \partial_{b)} H\right), \quad \delta B_{a b}=H^{-1} \phi^{c} \varepsilon_{a b c d} \partial^{d} H, \quad \delta \Phi=\frac{1}{2} H^{-2} \phi^{a} \partial_{a} H,
$$

we find that the variation of the DFT action results in

$$
\delta^{2} S_{\text {DFT,NSNS }}=2 \int \mathrm{d}^{2 D} X H^{-3} \partial_{a} H \partial_{b} H\left(\phi^{a} \square \phi^{b}\right) .
$$


Then the relevant integral results in

$$
\int_{\text {trans. }} \mathrm{d}^{4} x H^{-3} \partial_{a} H \partial_{b} H=\frac{\pi^{2} c_{2}}{2 c_{1}^{2}} \delta_{a b}
$$

giving a finite value for the harmonic function $H=c_{1}+c_{2} r^{-2}$. This implies that the scalar part of the effective action of the five-brane is given by the desired form:

$$
S \sim \int_{\mathrm{wv} .} \mathrm{d}^{6} x \phi^{a} \square \phi^{a} .
$$

For the five-branes of lower codimensions, the calculations are the same with the ones in the RR sector.

\subsection{Self-duality constraint}

Finally, we analyze the self-duality constraint of the $O(D, D)$ spinor $\chi$. Since we use the democratic formulation of the $\mathrm{RR}$ sector, we need to impose the self-duality constraint in order to rewrite the higher rank forms by the lower ones. The self-duality constraint is expressed as

$$
\not \partial \chi=-\mathcal{K} \not \partial \chi
$$

We derive the corresponding constraints for the fluctuations. By substituting the shift $\chi \rightarrow \chi_{0}+\delta \chi=0+\delta_{\lambda} \chi$ into the self-duality constraint, we have the following relation (the detailed calculations are found in appendix B)

$$
\begin{aligned}
& \sum_{p=0}^{6} \frac{1}{(6-p) !} \varepsilon_{n m_{1} \cdots m_{p-1} k_{1} \cdots k_{6-p}} \eta_{k_{1} l_{1}} \cdots \eta_{k_{6-p} l_{6-p}} F_{n m_{1} \cdots m_{p-1}} \psi^{l_{1} \cdots l_{6-p}}=-t \sum_{p=0}^{6} F_{l_{1} \cdots l_{p}} \psi^{l_{1} \cdots l_{p}} \\
& \sum_{p=0}^{6} \frac{1}{(6-p) !} \varepsilon_{n m_{1} \cdots m_{p-1} k_{1} \cdots k_{6-p}} \eta_{k_{1} l_{1}} \cdots \eta_{k_{6-p} l_{6-p}} F_{n m_{1} \cdots m_{p-1}} \psi^{l_{1} \cdots l_{6-p}}=-\frac{1}{t} \sum_{p=0}^{6} F_{l_{1} \cdots l_{p}} \psi^{l_{1} \cdots l_{p}} .
\end{aligned}
$$

We find that these conditions are consistent when $t=-1$ which precisely coincides with the result in the previous section. For $t=-1$, these conditions are rewritten as

$$
F_{m_{1} \cdots m_{6-p}}=\frac{(-)^{\frac{(6-p)(6-p-1)}{2}}}{(6-p) !} \varepsilon_{m_{1} \cdots m_{6-p}}^{n_{1} \cdots n_{p}} F_{n_{1} \cdots n_{p}} .
$$

Here $\varepsilon$ is the Levi-Civita symbol in six dimensions. The indices are raised and lowered by the six-dimensional Lorentz metric $\eta^{m n}$. We denote the condition (4.27) as

$$
F^{(p-6)}= \pm *_{6} F^{(p)} .
$$

This is nothing but the self-duality relation for the zero-modes in six dimensions. Now we write down the condition (4.28) explicitly in cases of type IIA and IIB supergravities. 
Type IIA case. For the type IIA case, the relevant RR potentials are

$$
C^{(1)}, C^{(3)}, C^{(5)}, C^{(7)}, C^{(9)} \text {. }
$$

The corresponding gauge transformations are given by

$$
\delta C^{(1)}=\mathrm{d} \lambda^{(0)}, \quad \delta C^{(3)}=\mathrm{d} \lambda^{(2)}, \quad \delta C^{(5)}=\mathrm{d} \lambda^{(4)}, \quad \delta C^{(7)}=\mathrm{d} \lambda^{(6)}, \quad \delta C^{(9)}=\mathrm{d} \lambda^{(8)} .
$$

Since the zero-modes associated with the RR gauge transformations are defined in the six-dimensional world-volume, we have $\lambda^{(8)}=\mathrm{d} \lambda^{(6)}=0$. Therefore, the non-zero field strengths for the zero-modes are given by

$$
F^{(1)}, \quad F^{(3)}, \quad F^{(5)} .
$$

Here $F^{(p+1)}=\mathrm{d} \lambda^{(p)}$ and the exterior derivative is defined in the six-dimensional worldvolume. The self-duality relations (4.28) are therefore

$$
F^{(1)}=-*_{6} F^{(5)}, \quad F^{(3)}=-*_{6} F^{(3)}, \quad F^{(5)}=-*_{6} F^{(1)} .
$$

The first and the last ones state that the degrees of freedom by $\lambda^{(4)}$ are given by $\lambda^{(0)}$. The second one implies that the 2 -form $\lambda^{(2)}$ is anti-self-dual (ASD). Therefore, the net zero-modes are given by

$$
\lambda^{(0)} \text { (scalar), } \quad \lambda^{(2)} \text { (ASD 2-form). }
$$

These together with the fluctuation zero-modes $\phi^{a},(a=6,7,8,9)$ precisely give the bosonic components of the six-dimensional $\mathcal{N}=(2,0)$ tensor multiplet.

Type IIB case. For the type IIA case, the relevant RR potentials are given by

$$
C^{(0)}, C^{(2)}, C^{(4)}, C^{(6)}, C^{(8)}, C^{(10)} .
$$

The corresponding gauge transformations are

$$
\begin{array}{llrl}
\delta C^{(0)}=0, & \delta C^{(2)}=\mathrm{d} \lambda^{(1)}, & \delta C^{(4)}=\mathrm{d} \lambda^{(3)}, \\
\delta C^{(6)}=\mathrm{d} \lambda^{(5)}, & \delta C^{(8)}=\mathrm{d} \lambda^{(7)}, & \delta C^{(10)}=\mathrm{d} \lambda^{(9)} .
\end{array}
$$

The RR 0-form is gauge invariant. Since $\lambda^{(p)}$ are defined in the six-dimensional worldvolume, we have $\lambda^{(7)}=\lambda^{(9)}=0$. Therefore the field strengths of the zero-modes are given by

$$
F^{(2)}, \quad F^{(4)}, \quad F^{(6)} .
$$

The self-duality relations (4.28) read

$$
F^{(2)}=*_{6} F^{(4)}, \quad F^{(4)}=*_{6} F^{(2)}, \quad F^{(6)}=0 .
$$

The first and the second ones state that the degrees of freedoms of $\lambda^{(3)}$ are given by $\lambda^{(1)}$. The last one implies $\lambda^{(5)}$ is non-dynamical in the five-brane world-volume. Therefore the net zero-mode is

$$
\lambda^{(1)} \text { (vector). }
$$

This together with the fluctuation zero-modes $\phi^{a},(a=6,7,8,9)$ precisely give the bosonic components of the six-dimensional $\mathcal{N}=(1,1)$ vector multiplet. 


\begin{tabular}{|l|l|c|c|c|}
\hline 6d supermultiplets & \multicolumn{4}{|c|}{ Five-branes } \\
\hline $\mathcal{N}=(2,0)$ tensor & IIA & NS5, Q5, SF5 & IIB & KKM, R5 \\
\hline $\mathcal{N}=(1,1)$ vector & IIA & KKM, R5 & IIB & NS5, Q5, SF5 \\
\hline
\end{tabular}

Table 1. The five-branes in type II theories and the corresponding supermultiplets in the worldvolumes. The KKM and SF5 stand for the KK-monopole and the space-filling five-brane.

\section{Effective theories of locally non-geometric five-branes}

In this section, we examine the world-volume effective theories of the five-branes in type II string theories. In particular, we focus on the effective theories of locally non-geometric five-branes. In the previous sections, we have determined the zero-modes of the five-branes. There are the four fluctuation zero-modes $\phi^{a}(a=6,7,8,9)$ and the tensor zero-modes in the RR sector. There are two classes of the tensor zero-modes, namely, the 1-form $\lambda^{(1)}$ and a pair of the scalar and the anti-self-dual 2-form $\left(\lambda^{(0)}, \lambda^{(2)}\right)$ depending on the possible $R R$ potentials in type IIA and IIB supergravities. They satisfy the desired field equations in the world-volumes and are organized into the bosonic sectors of the six-dimensional $\mathcal{N}=(1,1)$ vector and the $\mathcal{N}=(2,0)$ tensor multiplets. These multiplets and the corresponding five-branes are summarized in table 1.

We now examine the physical meaning of these zero-modes in each brane. Before that, we note that there are two kinds of locally non-geometric objects in string theory. One is the R-brane type that appear in table 1, the other is the localized KK-monopole type [21]. We first consider the R-brane type and then introduce the localized KK-monopole type.

\section{$5.1 \quad$ R-brane type}

The locally non-geometric objects of the R-brane type appear in the T-duality orbit including the ordinary geometric branes. In the following, we survey the effective theories of five-branes by starting from the familiar NS5-brane and applying the T-duality transformations.

NS5-brane and KK-monopole. For the type IIA NS5-brane, the world-volume theory is characterized by the six-dimensional $\mathcal{N}=(2,0)$ tensor multiplet. The 0 - and 2 -form fields in the tensor multiplet correspond to the zero-modes associated with the gauge transformations of the RR 1- and 3-forms. Among other things, the 0-form field in the type IIA NS5-brane world-volume is interpreted as the fluctuation along the M-circle. For the type IIB NS5-brane, the world-volume theory is governed by the six-dimensional $\mathcal{N}=(1,1)$ vector multiplet. The 1-form in the vector multiplet corresponds to the zero-mode associated with the gauge transformation of the RR 2-form.

For the zero-modes that come from the generalized Lie derivative, they are decomposed into those by the diffeomorphism $\delta=\mathcal{L}_{\zeta}$ and the $B$-field gauge transformation $\delta B=\mathrm{d} \Lambda$ whose parameters are given by

$$
\zeta^{a}=\epsilon^{a}=H^{-1} \phi^{a}(x), \quad \Lambda_{a}=\bar{\epsilon}_{a}=-H^{-1} \phi^{b}(x) b_{b a} .
$$


From this expression, the four scalar zero-modes $\phi^{a}$ in both of the NS5-branes are interpreted as the geometric fluctuations of branes in the transverse directions. Compared with the D-branes [45], one notices that the modes $\phi^{a}$ necessarily present in the $B$-field gauge transformation in the NS5-branes.

For the KK-monopoles, the transverse directions are given by the Taub-NUT space which accommodates one isometry direction. The T-duality transformation of the RR potentials increases or decreases their ranks. We are interested in the RR potentials whose indices extend to the world-volume directions of the five-branes. The T-duality transformation of the RR $p$-form along the $y^{9}$-direction results in the $(p+1)$-form whose increased index is given by $a=9$. Correspondingly, the zero-modes of the NS5-branes associated with the gauge transformations of the $\mathrm{RR}$ potentials become those with the increased index given by the isometry direction $(a=9)$. Therefore, the 0 - and the 2 -form zero-modes in the world-volume of the IIB KK-monopole stem from the gauge transformations of the RR 2 - and 4-forms whose gauge parameters contain the index $a=9$. The same is true for the type IIA KK-monopole.

One of the four geometric zero-modes in the NS5-brane is T-dualized and it turns out to be zero-modes associated with the gauge transformation of the NSNS $B$-field. Explicitly, this is given by the parameters

$$
\Lambda_{a}=\left(\Lambda_{i}, \Lambda_{9}\right)=\left(\bar{\epsilon}_{i}, \epsilon^{9}\right)=\left(H^{-1} \phi^{9} A_{i}, H^{-1} \phi^{9}\right), \quad(i=6,7,8) .
$$

Then we find that the corresponding gauge transformation becomes

$$
\delta B=\mathrm{d} \Lambda=\phi^{9} \eta,
$$

where we have introduced the basis $\eta$ defined by

$$
\eta=H^{-1} \mathrm{~d} A+\mathrm{d} H^{-1} \wedge\left(\mathrm{d} \bar{y}_{9}+A\right) .
$$

One finds that this basis satisfies the self-duality condition in the transverse fourdimensional Taub-NUT space

$$
\eta=*_{4} \eta
$$

This is consistent with the known results $[46,47]$. These zero-modes are summarized in table 2 .

Q5-brane. For the Q5-brane, there are two isometry directions to the transverse space. Only the two fluctuation zero-modes correspond to the geometric modes in the transverse directions. Two of the four fluctuation zero-modes in the NS5-brane are T-dualized giving rise to the two independent $B$-field gauge transformations. These are explicitly given by

$$
\delta B=\phi^{8} \mathrm{~d} H^{-1} \wedge \mathrm{d} \bar{y}_{8}+\phi^{9} \mathrm{~d} H^{-1} \wedge \mathrm{d} \bar{y}_{9} .
$$

This is a generalization of the $B$-field gauge zero-mode (5.3) in the Taub-NUT space. Conversely, the modes of the $B$-field gauge transformation in the isometric direction enters into 


\begin{tabular}{|l|c|c|c|c|}
\hline & $\phi^{6}, \phi^{7}, \phi^{8}$ & $\phi^{9}$ & 0 -form & 2 -form \\
\hline IIA NS5 & geometric & geometric & $\delta C^{(1)}=\mathrm{d} \lambda^{(0)}: \lambda^{(0)}$ & $\delta C^{(3)}=\mathrm{d} \lambda^{(2)}: \lambda_{m n}^{(2)}$ \\
\hline IIB KKM & geometric & self-dual $\delta B$ & $\delta C^{(2)}=\mathrm{d} \lambda^{(1)}: \lambda_{9}^{(1)}$ & $\delta C^{(4)}=\mathrm{d} \lambda^{(3)}: \lambda_{m n 9}^{(3)}$ \\
\hline & & & \multicolumn{2}{|c|}{1 -form } \\
\hline IIB NS5 & geometric & geometric & \multicolumn{2}{|c|}{$\delta C^{(2)}=\mathrm{d} \lambda^{(1)}: \lambda_{m}^{(1)}$} \\
\hline IIA KKM & geometric & self-dual $\delta B$ & \multicolumn{2}{|c|}{$\delta C^{(3)}=\mathrm{d} \lambda^{(2)}: \lambda_{m 9}^{(2)}$} \\
\hline
\end{tabular}

Table 2. Physical interpretations of the zero-modes in the six-dimensional $\mathcal{N}=(2,0)$ tensor and the $\mathcal{N}=(1,1)$ vector multiplets. The cases of the NS5-brane and the KK-monopole. The index structures of the gauge parameters are specified explicitly.

the corresponding diffeomorphism by the T-duality. This is a remnant of the mixing of the diffeomorphism and gauge parameters (5.1). Since the zero-modes of branes are determined by the local geometry, even though the Q-brane shows the globally non-geometric nature, they are still understandable in the sense of the ordinary space-time picture. In the effective action of the Q5-brane, the two geometric modes along the isometry directions disappear in the pull-backs of the background fields while the two modes in (5.6) show up as extra scalar fields $[35,36,39]$. This is consistent with our result.

As in the cases of the KK-monopoles, the tensor zero-modes appear from the higher ranks of the RR potentials whose indices extend along the isometry directions.

R5- and space-filling 5-branes. The above picture of the zero-modes becomes obscure when we try to understand locally non-geometric objects like the R-branes. For the R5brane, the solution loses the conventional geometric meaning since it inevitably involves the winding coordinates. There are two isometry directions in the R5-brane solution, namely, the $\bar{y}_{8^{-}}$and $\bar{y}_{9}$-directions in (2.25). Corresponding to these directions, there are two independent zero-modes associated with the $B$-field gauge transformations. Since the R5-brane is a domain wall type object in the conventional space-time, there is one geometric fluctuation mode along the $y^{6}$-direction. The last mode is given by the DFT gauge parameter $\xi_{7}=\Lambda_{7}=H^{-1} \phi^{7}$. Since this parameter gives the translational symmetry along the transverse direction to the R5-brane, it is natural to interpret it as the fluctuation in the winding space rather than the $B$-field gauge transformation. Since the R-brane dynamics is governed by the fluctuations along the winding space, it should play a role of a probe for winding space. This feature apparently distinguishes the R-brane from the conventional extended objects in string theory.

The same is true even for the space-filling branes. The solutions are localized in the winding space and spontaneously break the translational symmetry along these directions. Therefore, the two scalar fields on the space-filling brane (2.27) represent the fluctuations along the two winding directions. The other two are zero-modes associated with the $B$-field gauge transformations. If the two winding directions are smeared, the solution becomes a flat space. In this case, all the four fluctuation zero-modes correspond to the zero-modes by the $B$-field gauge transformations. 


\begin{tabular}{|l|c|c|c|c|c|c|}
\hline & $\phi^{6}$ & $\phi^{7}$ & $\phi^{8}$ & $\phi^{9}$ & 0 -form & 2 -form \\
\hline IIA Q5 & geometric & geometric & $\delta B$ & $\delta B$ & $\delta C^{(3)}=\mathrm{d} \lambda^{(2)}: \lambda_{89}^{(2)}$ & $\delta C^{(5)}=\mathrm{d} \lambda^{(4)}: \lambda_{m n 89}^{(4)}$ \\
\hline IIB R5 & geometric & winding & $\delta B$ & $\delta B$ & $\delta C^{(4)}=\mathrm{d} \lambda^{(3)}: \lambda_{789}^{(3)}$ & $\delta C^{(6)}=\mathrm{d} \lambda^{(5)}: \lambda_{m n 789}^{(5)}$ \\
\hline IIA SF5 & winding & winding & $\delta B$ & $\delta B$ & $\delta C^{(5)}=\mathrm{d} \lambda^{(4)}: \lambda_{6789}^{(4)}$ & $\delta C^{(7)}=\mathrm{d} \lambda^{(6)}: \lambda_{m n 6789}^{(6)}$ \\
\hline & & & & & \multicolumn{2}{|c|}{1 -form } \\
\hline IIB Q5 & geometric & geometric & $\delta B$ & $\delta B$ & \multicolumn{2}{|c|}{$\delta C^{(4)}=\mathrm{d} \lambda^{(3)}: \lambda_{m 89}^{(3)}$} \\
\hline IIA R5 & geometric & winding & $\delta B$ & $\delta B$ & \multicolumn{2}{|c|}{$\delta C^{(5)}=\mathrm{d} \lambda^{(4)}: \lambda_{m 789}^{(4)}$} \\
\hline IIB SF5 & winding & winding & $\delta B$ & $\delta B$ & \multicolumn{2}{|c|}{$\delta C^{(6)}=\mathrm{d} \lambda^{(5)}: \lambda_{m 6789}^{(5)}$} \\
\hline
\end{tabular}

Table 3. Physical interpretation of the zero-modes in the six-dimensional $\mathcal{N}=(2,0)$ tensor and the $\mathcal{N}=(1,1)$ vector multiplets. For the cases of the non-geometric Q5-, R5 and SF5-branes. The index structure of the gauge parameters are specified explicitly.

\begin{tabular}{|c|ccccc|}
\hline & NS5 & KKM & Q5 & R5 & SF5 \\
\hline diffeomorphism $\zeta^{a}$ & $H^{-1} \phi^{a}$ & $\left(\begin{array}{c}H^{-1} \phi^{6} \\
H^{-1} \phi^{7} \\
H^{-1} \phi^{8} \\
0\end{array}\right)$ & $\left(\begin{array}{c}H^{-1} \phi^{6} \\
H^{-1} \phi^{7} \\
H^{-1} \phi^{9} A_{8} \\
0\end{array}\right)$ & $\left(\begin{array}{c}H^{-1} \phi^{6} \\
0 \\
H^{-1} \phi^{9} A_{8} \\
0\end{array}\right)$ & $\left(\begin{array}{c}0 \\
0 \\
H^{-1} \phi^{9} A_{8} \\
0\end{array}\right)$ \\
gauge parameter $\Lambda_{a}$ & $H^{-1} b_{a b} \phi^{b}$ & $\left(\begin{array}{c}H^{-1} \phi^{9} A_{6} \\
H^{-1} \phi^{9} A_{7} \\
H^{-1} \phi^{9} A_{8} \\
H^{-1} \phi^{9}\end{array}\right)$ & $\left(\begin{array}{c}0 \\
0 \\
H^{-1} \phi^{8} \\
H^{-1} \phi^{9}\end{array}\right)$ & $\left(\begin{array}{c}0 \\
H^{-1} \phi^{7} \\
H^{-1} \phi^{8} \\
H^{-1} \phi^{9}\end{array}\right)$ & $\left(\begin{array}{c}H^{-1} \phi^{6} \\
H^{-1} \phi^{7} \\
H^{-1} \phi^{8} \\
H^{-1} \phi^{9}\end{array}\right)$ \\
\hline
\end{tabular}

Table 4. The diffeomorphism and the $B$-field gauge parameters in each T-duality frame.

All the zero-modes of the non-geometric branes are summarized in table 3 . The corresponding gauge parameters in DFT are summarized in table 4.

\subsection{Localized KK-monopole type}

Another type of the locally non-geometric object is the so-called localized KK-monopole solution. This is first proposed as a genuine T-duality counterpart of the NS5-brane [26]. Remember that when one relates the NS5-brane and the KK-monopole by the T-duality transformation, one needs to introduce the isometry along the transverse direction to the brane. This is achieved by performing the smearing in the NS5-brane solution and the resulting geometry is known as the H-monopole of codimension three. The isometry in the H-monopole corresponds to that of the Taub-NUT space in the T-dualized KK-monopole side. On the other hand, it was shown that the isometry in the H-monopole is broken by the string worldsheet instanton effects [27]. The original NS5-brane geometry is recovered by summing up all the instanton effects which are interpreted as the KK-modes in the geometry. The same phenomenon occurs in the KK-monopole side. The isometry of the Taub-NUT space is again broken by the worldsheet instanton effects [28] and the geometry is modified due to the T-dual of the KK-modes, namely, the string winding modes. 


\begin{tabular}{|c|ccccc|}
\hline & NS5 & KKw1 & Q5w2 & R5w3 & SF5w4 \\
\hline diffeomorphism $\zeta^{a}$ & \multirow{2}{*}{$H^{-1} \phi^{a}$} & $\left(\begin{array}{c}H^{-1} \phi^{6} \\
H^{-1} \phi^{7} \\
H^{-1} \phi^{8} \\
H^{-1} b_{9 b} \phi^{b}\end{array}\right)$ & $\left(\begin{array}{c}H^{-1} \phi^{6} \\
H^{-1} \phi^{7} \\
H^{-1} b_{8 b} \phi^{b} \\
H^{-1} b_{9 b} \phi^{b}\end{array}\right)$ & $\left(\begin{array}{c}H^{-1} \phi^{6} \\
H^{-1} b_{7 b} \phi^{b} \\
H^{-1} b_{8 b} \phi^{b} \\
H^{-1} b_{9 b} \phi^{b}\end{array}\right)$ & $\left(\begin{array}{c}H^{-1} b_{6 b} \phi^{b} \\
H^{-1} b_{7 b} \phi^{b} \\
H^{-1} b_{8 b} \phi^{b} \\
H^{-1} b_{9 b} \phi^{b}\end{array}\right)$ \\
gauge parameter $\Lambda_{a}$ & $H^{-1} b_{a b} \phi^{b}$ & $\left(\begin{array}{c}H^{-1} b_{6 b} \phi^{b} \\
H^{-1} b_{7 b} \phi^{b} \\
H^{-1} b_{8 b} \phi^{b} \\
H^{-1} \phi^{9}\end{array}\right)$ & $\left(\begin{array}{c}H^{-1} b_{6 b} \phi^{b} \\
H^{-1} b_{7 b} \phi^{b} \\
H^{-1} \phi^{8} \\
H^{-1} \phi^{9}\end{array}\right)$ & $\left(\begin{array}{c}H^{-1} b_{6 b} \phi^{b} \\
H^{-1} \phi^{7} \\
H^{-1} \phi^{8} \\
H^{-1} \phi^{9}\end{array}\right)$ & $\left(\begin{array}{c}H^{-1} \phi^{6} \\
H^{-1} \phi^{7} \\
H^{-1} \phi^{8} \\
H^{-1} \phi^{9}\end{array}\right)$ \\
\hline
\end{tabular}

Table 5. The diffeomorphism and the $B$-field gauge parameters in the localized five-branes.

As a result, the modified geometry of the KK-monopole is characterized by the winding coordinate $\tilde{x}$ and it ceases to be a solution to supergravity. This is known as the localized KK-monopole solution. Although it is not a solution to supergravity, it is in fact a solution to DFT [21]. This result carries over to the Q5-brane. The worldsheet instanton effects break the isometries in the Q5-brane geometry and they introduce the winding coordinate dependence to the Q5-brane geometry [30,31]. The modified Q5-brane is no longer a solution to supergravity but it should be a solution to DFT. We call this the localized Q5-brane. Indeed, these localized solutions are obtained by the formal T-duality transformations of the NS5-brane geometry without isometries. One notices that all the five-brane solutions discussed in section 2 are written down by applying the formal T-duality transformations to the NS5-brane irrespective of their codimensions. Once a T-duality transformation is applied, one transverse geometric coordinate (that corresponds to the Fourier conjugate of the KK-modes) is switched to the winding coordinate corresponding to the Fourier conjugate of the winding modes. We therefore obtain the localized KK-monopole (KKw1: KK-monopole with one winding coordinate dependence), the localized Q5-brane (Q5w2: Q5-brane with two winding coordinate dependence), the localized R5-brane (R5w3) and the localized SF5-brane (SF5w4). They are apparently locally non-geometric objects and are indeed the solutions to DFT [23].

It is obvious that our calculations determining the zero-modes are irrelevant to the explicit form of the harmonic function $H$. Only we required is that it satisfies the Laplace equation $\square H=0$ in the transverse directions. Therefore all the analysis discussed above are also applied to the localized solutions. The effective theories of these localized fivebranes are governed by the six-dimensional $\mathcal{N}=(2,0)$ tensor and the $\mathcal{N}=(1,1)$ vector multiplets. The corresponding gauge parameters in DFT are summarized in table 5. Again, a natural interpretation is that the modes given by $H^{-1} \phi^{a}$ are fluctuations along the winding directions rather than the $B$-field gauge transformations.

\section{Conclusion and discussions}

In this paper, we studied the world-volume effective theories of the five-branes in type II string theories within the formalism of double field theory (DFT). These include the NS5- 
brane, the KK-monopole, the Q5-, R5-branes and the space-filling brane. They appear in the T-duality orbits including the familiar NS5-branes. Among other things, the R5- and the space-filling branes are kinds of the locally non-geometric objects. Although they are no longer solutions to conventional supergravity, they are solutions to DFT.

We first determined the precise zero-modes associated with the spontaneous breaking of the translational symmetry in the doubled space. This symmetry is a part of the gauge symmetry in DFT given by the generalized Lie derivative. We showed that there are four bosonic zero-modes that correspond to the broken shift symmetry along the transverse directions to the five-branes and they satisfy the Klein-Gordon equation in the sixdimensional world-volume. For the five-branes that are solutions to supergravity, namely, for the ordinary geometric and the globally non-geometric branes, we demonstrated that parts of the scalar zero-modes are interpreted as transverse geometric fluctuation of branes. The remaining zero-modes come from the $B$-field gauge transformations. Compared with these supergravity branes, the R5- and the space-filling branes, have fluctuation zero-modes along the winding directions. Indeed, this interpretation is necessary for the locally nongeometric objects since their geometries are intrinsically characterized in the winding space.

We next determined the zero-modes associated with the gauge symmetry in the RR sector. They include the 1-form and the pair of the 0 -form and the self-dual 2-form. We find that these zero-modes are normalizable and they satisfy the Maxwell equations for $p$-forms in the world-volume. The four fluctuation scalar modes together with these RR zero-modes are organized into the bosonic sectors of the six-dimensional $\mathcal{N}=(1,1)$ vector and the $\mathcal{N}=(2,0)$ tensor multiplets.

We also studied the other type of locally non-geometric objects in string theory. They are branes of the so-called localized KK-monopole type. When we discuss the genuine T-dualized object of the localized, the non-smeared NS5-brane (not the H-monopole), it is inevitable to consider the localized KK-monopole in the winding space. The geometry of the localized KK-monopole is characterized by the winding coordinate and it is interpreted as the string worldsheet instanton effects. The notion of the localized KK-monopole is generalized to those for the Q-, R- and the space-filling branes. Their explicit solutions are written down in the winding space. These localized objects are not solutions to supergravity but are shown to be solutions to DFT [23]. We determined the zero-modes of these localized solutions and find that they are governed by the six-dimensional supermultiplets. Although the solutions of the locally non-geometric five-branes are given in the winding space, we note that their dynamics are governed by the conventional field theories described by the six-dimensional supermultiplets.

We stress that the notion of the locally non-geometric objects in the winding space is necessary for uncovering the nature of stringy geometries. Indeed, they never give rise in conventional supergravity which is based on the point particle (or the zero-slope limit of string) picture of geometries. DFT is one of a useful formalism to capture stringy geometries with their non-Riemannian structures [48-50]. A mathematically rigorous treatment of the doubled space would help us to understand the nature of the winding geometries [51-53].

We showed that the effective theories of the five-branes are given by the bosonic sectors of the six-dimensional $\mathcal{N}=(2,0)$ tensor and the $\mathcal{N}=(1,1)$ vector multiplets. Since the 
supergravity five-branes discussed in this paper are the half BPS objects preserving 16 supercharges, we expect that the locally non-geometric five-branes keep also the same supercharges. It is interesting to determine the fermionic zero-modes associated with the spontaneous breaking of supersymmetry in DFT $[54,55]$. It is also interesting to study the other locally non-geometric branes. For example, the U-duality generalization of DFT, known as exceptional field theory (EFT), involves many kinds of non-geometric objects [56-60]. We would come back to these issues in future studies.

\section{Acknowledgments}

The work of K.S. is supported by Grant-in-Aid for JSPS Research Fellow, JSPS KAKENHI Grant Number JP20J13957. The work of S.S. is supported in part by Grant-in-Aid for Scientific Research (C), JSPS KAKENHI Grant Number JP20K03952.

\section{A Quick introduction to double field theory}

We here introduce the brief summary of DFT. More details are found in [43, 61].

\section{A.1 NSNS sector}

Fields and gauge symmetries in the NSNS sector are geometrically unified in DFT. DFT is defined in the doubled space in which T-duality is realized manifestly. The doubled space is characterized by the coordinate given by

$$
X^{M}=\left(\begin{array}{c}
\tilde{x}_{\mu} \\
x^{\mu}
\end{array}\right)
$$

where $x^{\mu}$ is the Fourier conjugate to the Kaluza-Klein (KK) modes of a string, while $\tilde{x}_{\mu}$ is the conjugate to the string winding modes. The NSNS sector of type II supergravities is organized into an $O(D, D)$ tensor $\mathcal{H}_{M N}$ and a scalar $d$ known as the generalized metric and the generalized dilaton, respectively. We consider $D=10$ in the following.

These fields are parametrized as

$$
\mathcal{H}_{M N}=\left(\begin{array}{cc}
g^{\mu \nu} & -g^{\mu \rho} B_{\rho \nu} \\
B_{\mu \rho} g^{\rho \nu} & g_{\mu \nu}-B_{\mu \rho} g^{\rho \sigma} B_{\sigma \nu}
\end{array}\right), \quad e^{-2 d}=\sqrt{-g} e^{-2 \Phi} .
$$

The index of the $O(D, D)$ tensor is raised and lowered by the $O(D, D)$ invariant metric

$$
\eta_{M N}=\left(\begin{array}{cc}
0 & \delta^{\mu}{ }_{\nu} \\
\delta_{\mu}{ }^{\nu} & 0
\end{array}\right), \quad \eta^{M N}=\left(\begin{array}{cc}
0 & \delta_{\mu}{ }^{\nu} \\
\delta^{\mu}{ }_{\nu} & 0
\end{array}\right)
$$

The action of the NSNS sector in DFT is given by

$$
S_{\mathrm{DFT}, \mathrm{NS}}=\int \mathrm{d}^{2 D} X e^{-2 d} \mathcal{R}(\mathcal{H}, d),
$$


where the generalized Ricci scalar $\mathcal{R}$ is defined by

$$
\begin{aligned}
\mathcal{R}= & 4 \mathcal{H}^{M N} \partial_{M} \partial_{N} d-\partial_{M} \partial_{N} \mathcal{H}^{M N}-4 \mathcal{H}^{M N} \partial_{M} d \partial_{N} d+4 \partial_{M} \mathcal{H}^{M N} \partial_{N} d \\
& +\frac{1}{8} \mathcal{H}^{M N} \partial_{M} \mathcal{H}^{K L} \partial_{N} \mathcal{H}_{K L}-\frac{1}{2} \mathcal{H}^{M N} \partial_{M} \mathcal{H}^{K L} \partial_{K} \mathcal{H}_{N L} .
\end{aligned}
$$

This action is manifestly invariant under the $O(D, D)$ transformation. All the quantities in DFT should satisfy the physical condition $\eta^{M N} \partial_{M} \partial_{N} *=0$. One can show that the DFT action (A.4) is invariant under the following DFT gauge transformations:

$$
\begin{aligned}
\delta_{\xi} \mathcal{H}_{M N} & =\widehat{\mathcal{L}}_{\xi} \mathcal{H}_{M N}=\xi^{P} \partial_{P} \mathcal{H}^{M N}+\left(\partial^{M} \xi_{P}-\partial_{P} \xi^{M}\right) \mathcal{H}^{P N}+\left(\partial^{N} \xi_{P}-\partial_{P} \xi^{N}\right) \mathcal{H}^{M P} \\
\delta_{\xi} d & =\widehat{\mathcal{L}}_{\xi} d=\xi^{M} \partial_{M} d-\frac{1}{2} \partial_{M} \xi^{M}
\end{aligned}
$$

provided that the strong constraint

$$
\eta^{M N} \partial_{M} * \partial_{N} *=0
$$

is satisfied. One of the trivial solution to the strong constraint is that the derivative of the winding coordinates vanishes $\tilde{\partial}^{\mu} *=0$. With the imposition of the condition $\tilde{\partial}^{\mu} *=0$, the DFT action is reduced to the NSNS sector of type II supergravity:

$$
S_{\mathrm{DFT}, \mathrm{NS}} \stackrel{\tilde{\partial}^{\mu} *=0}{\longrightarrow} S_{\mathrm{SUGRA}, \mathrm{NS}}=\int \mathrm{d}^{D} x \sqrt{-g} e^{-2 \Phi}\left[R+4(\partial \Phi)^{2}-\frac{1}{12} H_{\mu \nu \rho} H^{\mu \nu \rho}\right] .
$$

\section{A.2 RR sector}

The RR sector of type II supergravities is described by an $O(D, D)$ Majorana spinor in DFT [42, 43, 62]. We introduce the gamma matrices $\Gamma^{M}$ satisfying the following Clifford algebra in $2 D$ dimensions:

$$
\left\{\Gamma^{M}, \Gamma^{N}\right\}=2 \eta^{M N} \mathbf{1}
$$

The gamma matrices $\Gamma^{M}=\left(\Gamma_{\mu}, \Gamma^{\mu}\right)$ are represented by

$$
\Gamma_{\mu}=\sqrt{2} \psi_{\mu}, \quad \Gamma^{\mu}=\sqrt{2} \psi^{\mu},
$$

where $\psi^{\mu}$ and $\psi_{\mu}$ are the fermionic creation and annihilation operators satisfying the following relations,

$$
\left\{\psi_{\mu}, \psi^{\nu}\right\}=\delta_{\mu}{ }^{\nu}, \quad\left\{\psi_{\mu}, \psi_{\nu}\right\}=0, \quad\left\{\psi^{\mu}, \psi^{\nu}\right\}=0, \quad\left(\psi_{\mu}\right)^{\dagger}=\psi^{\mu} .
$$

The Clifford vacuum $|0\rangle$ is defined by

$$
\psi_{\mu}|0\rangle=0, \quad\langle 0 \mid 0\rangle=1 .
$$

A general $O(D, D)$ spinor is expanded by the basis $\psi^{\mu}$. We consider a spinor $\chi$ whose component expansion is given by

$$
\chi=\sum_{p=0}^{D} \frac{1}{p !} C_{\mu_{1} \cdots \mu_{p}} \psi^{\mu_{1}} \cdots \psi^{\mu_{p}}|0\rangle .
$$

Here the coefficients $C_{\mu_{1} \cdots \mu_{p}}$ are identified with the RR $p$-forms. 
There is a group homomorphism $\rho: \operatorname{Pin}(D, D) \rightarrow O(D, D)$ such that $h=\rho(S)$ for $S \in \operatorname{Pin}(D, D), h \in O(D, D)$. This means we have the following relation

$$
S \Gamma_{M} S^{-1}=\Gamma_{N} h^{N}{ }_{M}
$$

Therefore $S$ is a spinor representation of $h$ satisfying $h \eta h^{T}=\eta$. Conversely, for any $h \in O(D, D)$, we have $\pm S \in \operatorname{Pin}(D, D)$.

One notices that a spinor in $\operatorname{Pin}(D, D)$ is decomposed into eigenstates $\chi_{ \pm}$associated with the eigenvalues $(-)^{N_{F}}= \pm 1$ :

$$
(-)^{N_{F}} \chi_{ \pm}=( \pm 1) \chi_{ \pm}
$$

Here, $N_{F}=\sum_{\mu} \psi^{\mu} \psi_{\mu}$ is the fermion number operator. Then, we have the following chiral spinors

$$
\begin{aligned}
& \chi_{+}=\left[\frac{1}{0 !} C^{(0)}+\frac{1}{2 !} C_{\mu_{1} \mu_{2}}^{(2)} \psi^{\mu_{1}} \psi^{\mu_{2}}+\cdots+\frac{1}{10 !} C_{\mu_{1} \cdots \mu_{10}}^{(10)} \psi^{\mu_{1}} \cdots \psi^{\mu_{10}}\right]|0\rangle \\
& \chi_{-}=\left[\frac{1}{1 !} C_{\mu_{1}}^{(1)} \psi^{\mu_{1}}+\frac{1}{3 !} C_{\mu_{1} \cdots \mu_{3}}^{(3)} \psi^{\mu_{1}} \cdots \psi^{\mu_{3}}+\cdots+\frac{1}{9 !} C_{\mu_{1} \cdots \mu_{9}}^{(9)} \psi^{\mu_{1}} \cdots \psi^{\mu_{9}}\right]|0\rangle .
\end{aligned}
$$

All the RR potentials in type IIA/IIB theories are incorporated in this formalism. This is nothing but the democratic formulation of type II supergravity. When we impose the chirality condition on the spinors, the $\operatorname{Pin}(D, D)$ symmetry is broken down to $\operatorname{Spin}(D, D)$ and we have either IIA or IIB theory.

The charge conjugation operator $C$ satisfying $C \Gamma^{M} C^{-1}=\left(\Gamma^{M}\right)^{\dagger}$ is defined by

$$
C=\left(\psi^{1}-\psi_{1}\right)\left(\psi^{2}-\psi_{2}\right) \cdots\left(\psi^{10}-\psi_{10}\right)
$$

This also satisfies the relations

$$
C \psi_{\mu} C^{-1}=\psi^{\mu}, \quad C \psi^{\mu} C^{-1}=\psi_{\mu} .
$$

A Dirac operator $\not \partial$ is defined by

$$
\not \partial=\frac{1}{\sqrt{2}} \Gamma^{M} \partial_{M}=\psi^{\mu} \partial_{\mu}+\psi_{\mu} \tilde{\partial}^{\mu} .
$$

This is an $O(D, D)$ invariant operator and nilpotent under the strong constraint:

$$
\not \partial^{2}=\frac{1}{4}\left\{\Gamma^{M}, \Gamma^{N}\right\} \partial_{M} \partial_{N}=\frac{1}{2} \eta^{M N} \partial_{M} \partial_{N}=0 .
$$

When we solve the strong constraint by imposing $\tilde{\partial}^{\mu} *=0$, the Dirac operator defines the field strengths of the RR potentials:

$$
\not \partial \chi=\sum_{p} \frac{1}{p !} \partial_{\mu} C_{\mu_{1} \cdots \mu_{p}} \psi^{\mu} \psi^{\mu_{1}} \cdots \psi^{\mu_{p}}|0\rangle .
$$

A spin representation $\mathbb{S}=\mathbb{S}^{\dagger} \in \operatorname{Spin}^{-}(10,10)$ of the generalized metric $\mathcal{H}$ is defined through the relation,

$$
\mathbb{S} \Gamma_{M} \mathbb{S}^{-1}=\Gamma_{N} \mathcal{H}^{N}{ }_{M}
$$


Here $\mathcal{H}^{N}{ }_{M}=\eta^{N P} \mathcal{H}_{P M}$ and $\mathcal{H}^{t}=\rho\left(\mathbb{S}^{\dagger}\right)=\mathcal{H}$. Then the DFT action in the RR sector is given by $[42,43]$,

$$
S_{\mathrm{DFT}, \mathrm{RR}}=\int \mathrm{d}^{2 D} X \frac{1}{4}(\not \partial \chi)^{\dagger} \mathbb{S}(\not \partial \chi) .
$$

Since the RR sector is introduced in the democratic formulation, we impose the following self-duality constraint to reduce the duplicated degrees of freedom:

$$
\not \partial \chi=-\mathcal{K} \not \partial \chi
$$

Here we have introduced $\mathcal{K}=C^{-1} \mathbb{S}$. The energy-momentum tensor for the matter sector is defined by

$$
\mathcal{E}^{M N}=-\frac{1}{16} \mathcal{H}^{\left(M_{P}\right.} \overline{\not \supset \chi} \Gamma^{N) P} \not \partial \chi
$$

Here $\bar{\chi}=\chi^{\dagger} C$ is the Dirac conjugation.

\section{B Detailed calculations}

Here we exhibit the detailed calculations that are skipped in the main text.

\section{B.1 Calculations on the generalized Ricci tensor}

Remember that the generalized Ricci tensor is given by $\mathcal{R}_{M N}=P_{M N}{ }^{K L} \mathcal{K}_{K L}$, we first decompose the $\mathcal{K}$ tensor. In the following, we solve the strong constraint by the condition $\bar{\partial}^{\mu} *=0$. By substituting the parametrization (3.3) of the generalized metric and dilaton, we find the following result:

$$
\begin{aligned}
\mathcal{K}_{\mu \nu}= & 2 \partial_{\mu} \nabla_{\nu} \Phi-\Gamma_{\mu \nu}^{\sigma} \nabla_{\sigma} \Phi+\left(B_{(\nu \mid \kappa} g^{\kappa \tau}\right)\left(g^{\rho \sigma} H_{\mid \mu) \tau \rho} \nabla_{\sigma} \Phi\right)+\left(B_{\mu \tau} g^{\tau \alpha}\right)\left(B_{\nu \kappa} g^{\kappa \beta}\right)\left(\Gamma_{\alpha \beta}^{\sigma} \nabla_{\sigma} \Phi\right) \\
& -\frac{1}{4}\left(g^{\rho \tau} g^{\sigma \kappa} H_{(\mu \mid \sigma \rho} H_{\mid \nu) \kappa \tau}+2 g^{\rho \sigma} B_{(\nu \mid \kappa}\left(H_{\mid \mu) \tau \rho}+\partial_{\mid \mu)} B_{\tau \rho}\right) \partial_{\sigma} g^{\tau \kappa}\right) \\
- & \frac{1}{2}\left(B_{(\nu \mid \kappa} g^{\kappa \tau}\right)\left(\left(g^{\rho \sigma} \partial_{\sigma} H_{\mid \mu) \tau \rho}+H_{\mid \mu) \tau \rho} \partial_{\sigma} g^{\rho \sigma}+g^{\rho \sigma} H_{\mid \mu) \tau \rho} \Gamma_{\sigma}\right)\right. \\
& \left.\quad+2 g^{\alpha \beta} \Gamma_{\alpha \tau}^{\sigma} \partial_{\sigma} B_{\mid \mu) \beta}-\partial_{\sigma} B_{\tau \rho} \partial_{\mid \mu)} g^{\rho \sigma}\right) \\
& -\partial_{\mu} \Gamma_{\nu}+\frac{1}{2} \partial_{\sigma} \Gamma_{\mu \nu}^{\sigma}+\frac{1}{2} \Gamma_{\mu \nu}^{\sigma} \Gamma_{\sigma}-\frac{1}{2} g_{(\nu \mid \tau} \Gamma_{\rho \sigma}^{\tau} \partial_{\mid \mu)} g^{\rho \sigma} \\
& -\left(B_{(\nu \mid \kappa} g^{\kappa \tau}\right)\left(B_{\mid \mu) \beta} \Gamma_{\alpha \tau}^{\sigma} \partial_{\sigma} g^{\alpha \beta}\right)-\frac{1}{2}\left(B_{\mu \tau} g^{\tau \alpha}\right)\left(B_{\nu \kappa} g^{\kappa \beta}\right)\left(\partial_{\sigma} \Gamma_{\alpha \beta}^{\sigma}+\Gamma_{\alpha \beta}^{\sigma} \Gamma_{\sigma}\right) .
\end{aligned}
$$

Here the ordinary Christoffel symbol for $g$ is defined by $\Gamma_{\mu \nu}^{\rho}=\frac{1}{2} g^{\rho \sigma}\left(\partial_{\mu} g_{\nu \sigma}+\partial_{\nu} g_{\mu \sigma}-\partial_{\sigma} g_{\mu \nu}\right)$. Similarly, $\mathcal{K}_{\mu}{ }^{\nu}$ is decomposed as

$$
\begin{aligned}
\mathcal{K}_{\mu}{ }^{\nu}= & \frac{1}{2}\left(g^{\tau \nu} g^{\rho \sigma} H_{\rho \mu \tau} \nabla_{\sigma} \Phi\right)+\left(B_{\mu \tau} g^{\tau \alpha}\right)\left(\Gamma_{\alpha \beta}^{\sigma} g^{\beta \nu} \nabla_{\sigma} \Phi\right) \\
- & \frac{1}{4}\left(g^{\rho \sigma}\left(H_{\rho \mu \tau}+\partial_{\mu} B_{\tau \rho}\right) \partial_{\sigma} g^{\tau \nu}+g^{\tau \nu}\left(H_{\rho \mu \tau} \partial_{\sigma} g^{\rho \sigma}+g^{\rho \sigma} \partial_{\sigma} H_{\rho \mu \tau}+g^{\rho \sigma} H_{\rho \mu \tau} \Gamma_{\sigma}\right)\right. \\
& \left.\quad+2 g^{\tau \alpha} \Gamma_{\alpha \beta}^{\sigma} g^{\beta \nu} \partial_{\sigma} B_{\mu \tau}-g^{\nu \tau} \partial_{\mu} g^{\rho \sigma} \partial_{\sigma} B_{\tau \rho}\right) \\
- & \frac{1}{2}\left(B_{\mu \tau} \Gamma_{\alpha \beta}^{\sigma} g^{\beta \nu} \partial_{\sigma} g^{\tau \alpha}\right)-\frac{1}{2}\left(B_{\mu \tau} g^{\tau \alpha}\right)\left(\Gamma_{\alpha \beta}^{\sigma} \partial_{\sigma} g^{\beta \nu}+g^{\beta \nu} \partial_{\sigma} \Gamma_{\alpha \beta}^{\sigma}+\Gamma_{\alpha \beta}^{\sigma} g^{\beta \nu} \Gamma_{\sigma}\right) .
\end{aligned}
$$


Finally, $\mathcal{K}^{\mu \nu}$ is decomposed as

$$
\mathcal{K}^{\mu \nu}=g^{\mu \tau} g^{\nu \kappa} \Gamma_{\tau \kappa}^{\sigma} \nabla_{\sigma} \Phi-\frac{1}{2} g^{\mu \tau} g^{\nu \kappa} \Gamma_{\tau \kappa}^{\sigma} \Gamma_{\sigma}-\frac{1}{2}\left(2 g^{(\mu \mid \tau} \Gamma_{\tau \kappa}^{\sigma} \partial_{\sigma} g^{\mid \nu) \kappa}+g^{\mu \tau} g^{\nu \kappa} \partial_{\sigma} \Gamma_{\tau \kappa}^{\sigma}\right) .
$$

By using these expressions and the explicit form of the projector $P_{M N}{ }^{K L}$, the generalized Ricci tensor is written in the following forms:

$$
\begin{aligned}
\mathcal{R}_{\mu \nu} & =\frac{1}{2}(g \text { eq. })_{\mu \nu}-\left(B g^{-1}\right)_{\left(\left.\mu\right|^{\beta}\right.}(B \text { eq. })_{\mid \nu) \beta}-\frac{1}{2}\left(B g^{-1}\right)_{\mu}{ }^{\alpha}\left(B g^{-1}\right)_{\nu}{ }^{\beta}(g \text { eq. })_{\alpha \beta}, \\
\mathcal{R}_{\mu}{ }^{\nu} & \left.=-\frac{1}{2} g^{\nu \beta}(B \text { eq. })_{\mu \beta}-\frac{1}{2}\left(B g^{-1}\right)_{\mu}{ }^{\alpha} g^{\nu \beta} \text { (g eq. }\right)_{\alpha \beta}, \\
\mathcal{R}^{\mu \nu} & \left.=-\frac{1}{2} g^{\mu \alpha} g^{\nu \beta} \text { (g eq. }\right)_{\alpha \beta},
\end{aligned}
$$

where we have defined the following expressions:

$$
\begin{aligned}
\text { (g eq. })_{\mu \nu} & =R_{\mu \nu}-\frac{1}{4} H_{\mu \rho \sigma} H_{\nu}^{\rho \sigma}+2 \nabla_{\mu} \nabla_{\nu} \Phi, \\
(B \text { eq. })_{\mu \nu} & =\frac{1}{2} \nabla^{\alpha} H_{\alpha \mu \nu}-H_{\alpha \mu \nu} \nabla^{\alpha} \Phi \\
\left(B g^{-1}\right)_{\mu}{ }^{\nu} & =B_{\mu \rho} g^{\rho \nu} .
\end{aligned}
$$

\section{B.2 Calculations on the RR zero-modes}

With the imposition of the strong constraint and by substituting the ansatz (4.2) with the promoted field $\lambda$ into the equation of motion (4.6), we find the fluctuation satisfies

$$
\left(\psi^{a} \partial_{a}+\psi^{m} \partial_{m}\right)\left[C \mathbb{S}_{H}\left(\psi^{b} \partial_{b}+\psi^{n} \partial_{n}\right) \delta_{\lambda} \chi\right]=0,
$$

where the indices $m, n, \ldots=0,1, \ldots, 5$ and $a, b, \ldots=6, \ldots, 9$ run over the world-volume and the transverse directions. Here $H$ is the generalized metric associated with the localized DFT monopole (2.11). We first evaluate the derivatives on the fluctuation in the bracket:

$$
\begin{aligned}
\left(\psi^{b} \partial_{b}+\psi^{n} \partial_{n}\right) \delta_{\lambda} \chi= & \frac{1}{2} e^{\frac{1}{2} b \psi \psi} \partial_{b} b_{e^{\prime} f^{\prime}} \psi^{e^{\prime} f^{\prime}}\left[\frac{1}{2} H^{t} \partial_{c} b_{e f} \psi^{e f}+t H^{t-1} \partial_{c} H\right] \psi^{b c} \lambda \\
& +e^{\frac{1}{2} b \psi \psi}\left[\frac{1}{2} t H^{t-1} \partial_{b} H \partial_{c} b_{e f} \psi^{e f}+\frac{1}{2} H^{t} \partial_{b} \partial_{c} b_{e f} \psi^{e f}\right. \\
& \left.+t(t-1) H^{t-1} \partial_{b} H \partial_{c} H+t H^{t-1} \partial_{b} \partial_{c} H\right] \psi^{b c} \lambda \\
& -e^{\frac{1}{2} b \psi \psi}\left[\frac{1}{2} H^{t} \partial_{c} b_{e f} \psi^{e f}+t \partial_{c} H\right] \psi^{c} \psi^{n} \partial_{n} \lambda .
\end{aligned}
$$

Since the indices $a, b, c, \ldots$ run in the transverse directions to the five-brane, we have $\psi^{e^{\prime} f^{\prime} e f b c}=0$ due to the anti-symmetric nature of $\psi^{a}$. Then the first term in the bracket in the first line vanishes. By the factor $\psi^{b c}$, terms with the indices $b c$ are all anti-symmetrized. This simplifies the expression (B.7) and we have

$$
\begin{aligned}
(\mathrm{B} .7)= & \frac{1}{2} t e^{\frac{1}{2} b \psi \psi} H^{t-1} \partial_{c} H \partial_{b} b_{e f} \psi^{e f b c} \lambda+\frac{1}{2} t e^{\frac{1}{2} b \psi \psi} H^{t-1} \partial_{b} H \partial_{c} b_{e f} \psi^{e f b c} \lambda \\
& -\frac{1}{2} e^{\frac{1}{2} b \psi \psi} H^{s} \partial_{c} b_{e f} \sum_{p} \frac{1}{(p-1) !} \partial_{n} \lambda_{m_{1} \cdots m_{p-1}} \psi^{e f c} \psi^{n m_{1} \cdots m_{p-1}}|0\rangle \\
& -t e^{\frac{1}{2} b \psi \psi} H^{t-1} \partial_{c} H \sum_{p} \frac{1}{(p-1) !} \partial_{n} \lambda_{m_{1} \cdots m_{p-1}} \psi^{c} \psi^{n m_{1} \cdots m_{p-1}}|0\rangle .
\end{aligned}
$$


We next evaluate the term

$$
\mathbb{S}_{H}\left(\psi^{b} \partial_{b}+\psi^{n} \partial_{n}\right) \delta_{\lambda} \chi
$$

The spinor representation of the generalized metric is decomposed as

$$
\mathbb{S}_{H}=S_{B}^{\dagger} S_{g}^{-1} S_{B}, \quad S_{B}=e^{-\frac{1}{2} b_{a b} \psi^{a b}}, \quad S_{B}^{\dagger}=e^{\frac{1}{2} b_{a b} \psi_{a} \psi_{b}},
$$

where $S_{g}=S_{g}^{\dagger}$ is the spinor representation of $g_{\mu \nu}$. Using this expression we have

$$
\begin{aligned}
(\mathrm{B} .9)= & -\frac{1}{2} e^{\frac{1}{2} b_{a b} \psi_{a b}} S_{g}^{-1}\left[\frac{1}{2} H^{t} \partial_{c} b_{e f} \sum_{p} \frac{1}{(p-1) !} \partial_{n} \lambda_{m_{1} \cdots m_{p-1}} \psi^{e f c} \psi^{n m_{1} \cdots m_{p-1}}|0\rangle\right] \\
& -t e^{\frac{1}{2} b_{a b} \psi_{a b}} S_{g}^{-1}\left[H^{t-1} \partial_{c} H \sum_{p} \frac{1}{(p-1) !} \partial_{n} \lambda_{m_{1} \cdots m_{p-1}} \psi^{c} \psi^{n m_{1} \cdots m_{p-1}}|0\rangle\right]
\end{aligned}
$$

Note that the unconventional index contractions with the annihilation operator $\psi_{\mu}$. By using the formula [43]

$$
S_{g}^{-1} \psi^{\mu_{1}} \cdots \psi^{\mu_{p}}|0\rangle=-\sqrt{|\operatorname{det} g|} g^{\mu_{1} \nu_{1}} \cdots g^{\mu_{p} \nu_{p}} \psi^{\nu_{1}} \cdots \psi^{\nu_{p}}|0\rangle,
$$

and the localized DFT monopole solution (2.11), we find

$$
\begin{aligned}
S_{g}^{-1} \psi^{e f c} \psi^{n m_{1} \cdots m_{p-1}}|0\rangle=-\sqrt{|\operatorname{det} g| g^{e e^{\prime}}} g^{f f^{\prime}} g^{c c^{\prime}} g^{n l} g^{m_{1} n_{1}} \cdots g^{m_{p-1} n_{p-1}} \psi^{e^{\prime} f^{\prime} c^{\prime}} \psi^{l n_{1} \cdots n_{p-1}}|0\rangle \\
=-\sqrt{H^{4}} H^{-1} \delta^{e e^{\prime}} H^{-1} \delta^{f f^{\prime}} H^{-1} \delta^{c c^{\prime}} \eta^{n l} \eta^{m_{1} n_{1}} \cdots \eta^{m_{p-1} n_{p-1}} \psi^{e^{\prime} f^{\prime} c^{\prime}} \psi^{l n_{1} \cdots n_{p-1}}|0\rangle \\
=-H^{-1} \eta^{n l} \eta^{m_{1} n_{1}} \cdots \eta^{m_{p-1} n_{p-1}} \psi^{e f c} \psi^{l n_{1} \cdots n_{p-1}}|0\rangle .
\end{aligned}
$$

Likewise, we have

$$
S_{g}^{-1} \psi^{c} \psi^{n m_{1} \cdots m_{p-1}}|0\rangle=-H \eta^{l n} \eta^{m_{1} n_{1}} \cdots \eta^{m_{p-1} n_{p-1}} \psi^{c} \psi^{l n_{1} \cdots n_{p-1}}|0\rangle .
$$

Then,

$$
\begin{aligned}
(\mathrm{B} .11)= & \frac{1}{2} e^{\frac{1}{2} b_{a b} \psi_{a b}} H^{t-1} \partial_{c} b_{e f} \sum_{p} \frac{1}{(p-1) !} \partial_{n} \lambda_{m_{1} \cdots m_{p-1}} \eta^{n l} \eta^{m_{1} n_{1}} \cdots \eta^{m_{p-1} n_{p-1}} \psi^{e f c} \psi^{l n_{1} \cdots n_{p-1}}|0\rangle \\
& +t e^{\frac{1}{2} b_{a b} \psi_{a b}} H^{t} \partial_{c} H \sum_{p} \frac{1}{(p-1) !} \partial_{n} \lambda_{m_{1} \cdots m_{p-1}} \eta^{n l} \eta^{m_{1} n_{1}} \cdots \eta^{m_{p-1} n_{p-1}} \psi^{c} \psi^{l n_{1} \cdots n_{p-1}}|0\rangle \\
= & \frac{1}{2} e^{\frac{1}{2} b_{a b} \psi_{a b}} H^{t-1} \partial_{c} b_{e f} \sum_{p} f^{l n_{1} \cdots n_{p-1}} \psi^{e f c} \psi^{l n_{1} \cdots n_{p-1}}|0\rangle \\
& +t e^{\frac{1}{2} b_{a b} \psi_{a b}} H^{t} \partial_{c} H \sum_{p} f^{l n_{1} \cdots n_{p-1}} \psi^{c} \psi^{l n_{1} \cdots n_{p-1}}|0\rangle .
\end{aligned}
$$

Here we have defined

$$
f^{l n_{1} \cdots n_{p-1}}=\frac{1}{(p-1) !} \partial_{n} \lambda_{m_{1} \cdots m_{p-1}} \eta^{n l} \eta^{m_{1} n_{1}} \cdots \eta^{m_{p-1} n_{p-1}} .
$$

Finally, we evaluate the quantity

$$
\left(\psi^{a} \partial_{a}+\psi^{m} \partial_{m}\right)\left[C \mathbb{S}_{H}\left(\psi^{b} \partial_{b}+\psi^{n} \partial_{n}\right) \delta_{\lambda} \chi\right] .
$$


The charge conjugation operator $C$ satisfies $\psi^{\mu} C=C \psi_{\mu}$. Then, we have

$$
\begin{array}{r}
(\mathrm{B} .17)=C\left(\psi_{a} \partial_{a}+\psi_{m} \partial_{m}\right)\left[\frac{1}{2} e^{\frac{1}{2} b_{a b} \psi_{a b}} H^{t-1} \partial_{c} b_{e f} \sum_{p} f^{l n_{1} \cdots n_{p-1}} \psi^{e f c} \psi^{l n_{1} \cdots n_{p-1}}|0\rangle\right. \\
\left.+t e^{\frac{1}{2} b_{a b} \psi_{a b}} H^{t} \partial_{c} H \sum_{p} f^{l n_{1} \cdots n_{p-1}} \psi^{c} \psi^{l n_{1} \cdots n_{p-1}}|0\rangle\right] .
\end{array}
$$

Since all the annihilation operators are anti-commute with each other, we have

$$
\partial_{a} e^{\frac{1}{2} b_{a b} \psi_{a b}}=\frac{1}{2} \partial_{a} b_{e f} \psi_{e f} e^{\frac{1}{2} b_{a b} \psi_{a b}}=\frac{1}{2} e^{\frac{1}{2} b_{a b} \psi_{a b}} \partial_{a} b_{e f} \psi_{e f},
$$

and then we obtain

$$
\begin{aligned}
& (\mathrm{B} .18)=C \frac{1}{2} \psi_{a^{\prime}}\left[e^{\frac{1}{2} b_{a b} \psi_{a b}} \frac{1}{2} \partial_{a^{\prime}} b_{e^{\prime} f^{\prime}} \psi_{e^{\prime} f^{\prime}} \partial_{c} b_{e f} \cdot H^{t-1}+e^{\frac{1}{2} b_{a b} \psi_{a b}} \partial_{a^{\prime}} \partial_{c} b_{e f} \cdot H^{t-1}\right. \\
& \left.+e^{\frac{1}{2} b_{a b} \psi_{a b}} \partial_{c} b_{e f} \cdot(s-1) H^{t-2} \partial_{a^{\prime}} H\right] \sum_{p} f^{l n_{1} \cdots n_{p-1}} \psi^{e f c} \psi^{l n_{1} \cdots n_{p-1}}|0\rangle \\
& +C t \psi_{a^{\prime}}\left[e^{\frac{1}{2} b_{a b} \psi_{a b}} \frac{1}{2} \partial_{a^{\prime}} b_{e^{\prime} f^{\prime}} \psi_{e^{\prime} f^{\prime}} H^{t} \partial_{c} H+e^{\frac{1}{2} b_{a b} \psi_{a b}} \cdot t H^{t-1} \partial_{a^{\prime}} H \partial_{c} H\right. \\
& \left.+e^{\frac{1}{2} b_{a b} \psi_{a b}} H^{t} \partial_{a^{\prime}} \partial_{c} H\right] \sum_{p} f^{l n_{1} \cdots n_{p-1}} \psi^{c} \psi^{l n_{1} \cdots n_{p-1}}|0\rangle \\
& +C \frac{1}{2} \psi_{m}\left[e^{\frac{1}{2} b_{a b} \psi_{a b}} H^{t-1} \partial_{c} b_{e f}\right] \sum_{p} \partial_{m} f^{l n_{1} \cdots n_{p-1}} \psi^{e f c} \psi^{l n_{1} \cdots n_{p-1}}|0\rangle \\
& +C t \psi_{m}\left[e^{\frac{1}{2} b_{a b} \psi_{a b}} H^{t} \partial_{c} H\right] \sum_{p} \partial_{m} f^{l n_{1} \cdots n_{p-1}} \psi^{c} \psi^{l n_{1} \cdots n_{p-1}}|0\rangle .
\end{aligned}
$$

When the number of the creation and the annihilation operators are not balanced, we have the vanishing contribution like $\psi_{a} \psi_{e^{\prime} f^{\prime}} \psi^{c}|0\rangle=0$. Using this fact, we find

$$
\begin{aligned}
(\mathrm{B} .20)=C e^{\frac{1}{2} b_{a b} \psi_{a b}[}\left[\frac{1}{4} \partial_{a^{\prime}} b_{e^{\prime} f^{\prime}} \partial_{c} b_{e f} H^{t-1} \psi_{a^{\prime} e^{\prime} f^{\prime}} \psi^{e f c}+\frac{1}{2} \partial_{a^{\prime}} \partial_{c} b_{e f} \cdot H^{t-1} \psi_{a^{\prime}} \psi^{e f c}\right. \\
+\frac{1}{2}(t-1) \partial_{c} b_{e f} H^{t-2} \partial_{a^{\prime}} H \psi_{a^{\prime}} \psi^{e f c} \\
\left.+t^{2} H^{t-1} \partial_{a^{\prime}} H \partial_{c} H \psi_{a^{\prime}} \psi^{c}+t H^{t} \partial_{a^{\prime}} \partial_{c} H \psi_{a^{\prime}} \psi^{c}\right] \sum_{p} f^{l n_{1} \cdots n_{p-1}} \psi^{l n_{1} \cdots n_{n-p}}|0\rangle \\
+C e^{\frac{1}{2} b_{a b} \psi_{a b}}\left[-\frac{1}{2} H^{t-1} \partial_{c} b_{e f} \psi^{e f c}-t H^{t} \partial_{c} H \psi^{c}\right] \sum_{p} \partial_{m} f^{l n_{1} \cdots n_{p-1}} \psi_{m} \psi^{l n_{1} \cdots n_{p-1}}|0\rangle .
\end{aligned}
$$

The equation for zero-modes is therefore (B.21) $=0$. Since $\psi_{a}, \psi^{c}$ anti-commutes with $\psi^{m}$ and

$$
\psi_{a} \psi^{c}|0\rangle=\left(\delta_{a}^{c}-\psi^{c} \psi_{a}\right)|0\rangle=\delta_{a}{ }^{c}|0\rangle,
$$


we have

$$
t H^{t} \partial_{a} \partial_{c} H \psi_{a} \psi^{c}|0\rangle=t H^{t} \square H|0\rangle=0 .
$$

Here we have used the fact that $H$ is a harmonic function. On the other hand, by using $\left\{\psi^{a}, \psi_{b}\right\}=\delta^{a}{ }_{b}$ iteratively, we find

$$
\psi_{a} \psi^{e f c}|0\rangle=\left(\delta_{a}^{e} \psi^{f c}-\delta_{a}^{f} \psi^{e c}+\delta_{a}^{c} \psi^{e f}\right)|0\rangle .
$$

Then we find

$$
\frac{1}{2} \partial_{a} \partial_{c} b_{e f} \cdot H^{t-1} \psi_{a} \psi^{e f c}|0\rangle=\frac{1}{2} H^{t-1}\left(\partial_{a} \partial_{c} b_{a b}+\partial_{a} \partial_{b} b_{c a}+\square b_{b c}\right) \psi^{b c}|0\rangle .
$$

From the BPS condition for the DFT monopole (2.15), we have the relation,

$$
\left(\square b_{b c}+\partial_{a} \partial_{b} b_{c a}+\partial_{a} \partial_{c} b_{a b}\right)=\varepsilon_{a b c d} \partial_{a} \partial_{d} H=0 .
$$

Similarly, we have

$$
\begin{aligned}
& \frac{1}{2}(t-1) \partial_{c} b_{e f} H^{t-2} \partial_{a} H \psi_{a} \psi^{e f c}|0\rangle \\
= & \frac{1}{2}(t-1) H^{t-2} \partial_{[c} b_{e f]} \partial_{a} H \psi_{a} \psi^{e f c}|0\rangle \\
= & \frac{1}{2}(t-1) H^{t-2} \frac{1}{3} \varepsilon_{c e f d} \partial_{d} H \partial_{a} H\left(\delta_{a}{ }^{e} \psi^{f c}-\delta_{a}{ }^{f} \psi^{e c}+\delta_{a}{ }^{c} \psi^{e f}\right)|0\rangle \\
= & 0 .
\end{aligned}
$$

We have also

$$
t^{2} H^{t-1} \partial_{a} H \partial_{c} \psi_{a} \psi^{c}|0\rangle=t^{2} H^{t-1} \partial_{a} H \partial_{a} H|0\rangle
$$

Finally, by using the relation

$$
\begin{aligned}
\psi_{a b c} \psi^{d e f}|0\rangle & =-\left(\delta_{a}{ }^{d} \delta_{b}{ }^{e} \delta_{c}{ }^{f}-\delta_{a}{ }^{d} \delta_{b}{ }^{f} \delta_{c}{ }^{e}+\delta_{a}{ }^{e} \delta_{b}{ }^{f} \delta_{c}{ }^{d}-\delta_{a}{ }^{e} \delta_{b}{ }^{d} \delta_{c}{ }^{f}+\delta_{a}{ }^{f} \delta_{b}{ }^{d} \delta_{c}{ }^{e}-\delta_{a}{ }^{f} \delta_{b}{ }^{e} \delta_{c}{ }^{d}\right)|0\rangle \\
& =-3 ! \delta_{a}{ }^{[d} \delta_{b}{ }^{e} \delta_{c}{ }^{f]}|0\rangle
\end{aligned}
$$

we find

$$
\frac{1}{4} H^{t-1} \partial_{a} b_{b c} \partial_{d} b_{e f} \psi_{a b c} \psi^{d e f}|0\rangle=-H^{t-1} \partial_{a} H \partial_{a} H|0\rangle .
$$

Therefore, we find that the first and the second lines in (B.21) gives

$$
\left[-H^{t-1} \partial_{a} H \partial_{a} H+t^{2} H^{t-1} \partial_{a} H \partial_{a} H\right] \sum_{p} f^{l n_{1} \cdots n_{p-1}} \psi^{l n_{1} \cdots n_{p-1}}|0\rangle .
$$

From the expression in (B.21), this terms should vanish by itself. Therefore, we find,

$$
t= \pm 1
$$


On the other hand, by using $\left\{\psi_{i}, \psi^{j}\right\}=\delta_{i}{ }^{j}$ several times, we find

$$
\psi_{m} \psi^{l n_{1} \cdots n_{p-1}}|0\rangle=\left(\delta_{m}^{l} \psi^{n_{1} \cdots n_{p-1}}-\delta_{m}{ }^{n_{1}} \psi^{l n_{2} \cdots n_{p-1}}+\cdots+(-)^{p-1} \delta_{m}^{n_{p-1}} \psi^{l n_{1} \cdots n_{p-2}}\right)|0\rangle
$$

Then we find that the terms in the second bracket in (B.21) vanishes when

$$
\partial_{m_{1}} F^{m_{1} \cdots m_{p}}=0
$$

where we have defined

$$
F^{m_{1} m_{2} \cdots m_{p}}=p ! \partial^{\left[m_{1}\right.} \lambda^{\left.m_{2} \cdots m_{p}\right]}
$$

The condition (B.34) is nothing but the Maxwell equation for the $p$-forms.

\section{B.3 Calculations on the normalizability}

We here show the derivation of the equation (4.11). The derivative of the fluctuation results in

$$
\begin{aligned}
\not \partial \delta \chi= & -\frac{1}{2} e^{\frac{1}{2} b_{a b} \psi^{a b}} H^{t} \partial_{c} b_{e f} \sum_{p} f_{n m_{1} \cdots m_{p-1}} \psi^{e f c} \psi^{n m_{1} \cdots m_{p-1}}|0\rangle \\
& -t e^{\frac{1}{2} b_{a b} \psi^{a b}} H^{t-1} \partial_{c} H \sum_{p} f_{n m_{1} \cdots m_{p-1}} \psi^{c} \psi^{n m_{1} \cdots m_{p-1}}|0\rangle .
\end{aligned}
$$

By using the relation $\left(\psi^{\mu_{1}} \cdots \psi^{\mu_{p}}|0\rangle\right)^{\dagger}=\langle 0| \psi_{\mu_{p}} \cdots \psi_{\mu_{1}}$ and the fact $\left(e^{\frac{1}{2} b_{a b} \psi^{a b}}\right)^{\dagger}=e^{-\frac{1}{2} b_{a b} \psi_{a b}}$, we obtain

$$
\begin{aligned}
(\not \partial \delta \chi)^{\dagger}= & -\frac{1}{2} H^{t} \partial_{c} b_{e f} \sum_{p} f_{n m_{1} \cdots m_{p-1}}\langle 0| \psi_{m_{p-1} \cdots m_{1} n} \psi_{c f e} e^{-\frac{1}{2} b_{a b} \psi_{a b}} \\
& -t H^{t-1} \partial_{c} H \sum_{p} f_{n m_{1} \cdots m_{p-1}}\langle 0| \psi_{m_{p-1} \cdots m_{1} n} \psi_{c} e^{-\frac{1}{2} b_{a b} \psi_{a b}}
\end{aligned}
$$

Further, we have

$$
\begin{aligned}
\mathbb{S}_{H} \not \partial \delta \chi= & \frac{1}{2} e^{\frac{1}{2} b_{a b} \psi_{a b}} H^{t-1} \partial_{c} b_{e f} \sum_{p} f_{n \cdots m_{p-1}} \eta^{n l} \cdots \eta^{m_{p-1} n_{p-1}} \psi^{e f c} \psi^{l \cdots n_{p-1}}|0\rangle \\
& +t e^{\frac{1}{2} b_{a b} \psi_{a b}} H^{t} \partial_{c} H \sum_{p} f_{n \cdots m_{p-1}} \eta^{n l} \cdots \eta^{m_{p-1} n_{p-1}} \psi^{c} \psi^{l \cdots n_{p-1}}|0\rangle
\end{aligned}
$$

Then we find

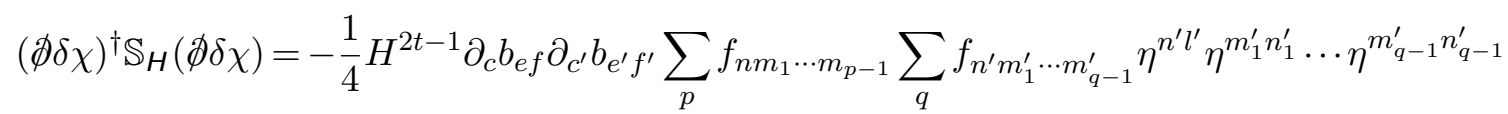

$$
\begin{aligned}
& \times\langle 0| \psi_{m_{p-1} \cdots m_{1}} \psi_{c f e} \psi^{e^{\prime} f^{\prime} c^{\prime}} \psi^{l^{\prime} n_{1}^{\prime} \cdots n_{q-1}^{\prime}|0\rangle} \\
& -t^{2} H^{2 t-1} \partial_{c} H \partial_{c^{\prime}} H \sum_{p} f_{n m_{1} \cdots m_{p-1}} \sum_{q} f_{n^{\prime} m_{1}^{\prime} \cdots m_{q-1}^{\prime}} \eta^{n^{\prime} l^{\prime}} \eta^{m_{1}^{\prime} n_{1}^{\prime} \cdots \eta^{m_{q-1}^{\prime}} n_{q-1}^{\prime}} \\
& \times\left\langle 0\left|\psi_{m_{p-1} \cdots m_{1}} \psi_{c} \psi^{c^{\prime}} \psi^{l^{\prime} n_{1}^{\prime} \cdots n_{q-1}^{\prime}}\right| 0\right\rangle .
\end{aligned}
$$




\section{B.4 Calculations on the self-duality constraint}

Here we show the derivation of the conditions (4.26). The self-duality constraint of the $O(D, D)$ spinor fluctuation $\delta_{\lambda} \chi$ is given by

$$
\not \partial \delta_{\lambda} \chi=C \mathbb{S}_{H}\left(\not \partial \delta_{\lambda} \chi\right)
$$

By using the relation $C \psi_{\mu}=\psi^{\mu} C, C S_{g}^{-1}=-S_{g} C$, we find

$$
C \mathbb{S}_{H}=C e^{\frac{1}{2} b_{a b} \psi_{a b}} S_{g}^{-1} e^{-\frac{1}{2} b_{c d} \psi^{c d}}=-e^{\frac{1}{2} b_{a b} \psi^{a b}} S_{g} C e^{-\frac{1}{2} b_{c d} \psi^{c d}} .
$$

Then the equation (B.40) becomes

$$
\not \partial \delta_{\lambda} \chi=-e^{\frac{1}{2} b_{a b} \psi^{a b}} S_{g} C e^{-\frac{1}{2} b_{c d} \psi^{c d}} \not \partial \delta_{\lambda} \chi .
$$

On the other hand, using the calculations in the previous subsection, the left-hand side in (B.42) becomes

$$
\begin{aligned}
\not \partial \delta_{\lambda} \chi= & -\frac{1}{6} e^{\frac{1}{2} b_{a b} \psi^{a b}} H^{t} \partial_{d} H \varepsilon_{c e f d} \sum_{p} F_{n m_{1} \cdots m_{p-1}} \psi^{c e f} \psi^{n m_{1} \cdots m_{p-1}}|0\rangle \\
& -t e^{\frac{1}{2} b_{a b} \psi^{a b}} H^{t-1} \partial_{c} H \sum_{p} F_{n m_{1} \cdots m_{p-1}} \psi^{c} \psi^{n m_{1} \cdots m_{p-1}}|0\rangle .
\end{aligned}
$$

Here we have used $\partial_{[a} b_{b c]}=\frac{1}{3} \varepsilon_{a b c d} \partial_{d} H$ and introduced $F_{\mu_{1} \mu_{2} \cdots \mu_{p}}=p ! \partial_{\left[\mu_{1}\right.} \lambda_{\left.\mu_{2} \cdots \mu_{p}\right]}$. The right-hand side in (B.42) is calculated as

$$
\begin{aligned}
-e^{\frac{1}{2} b_{a b} \psi^{a b}} S_{g} C e^{-\frac{1}{2} b_{c d} \psi^{c d}} \not \partial \delta_{\lambda} \chi= & \frac{1}{6} e^{\frac{1}{2} b_{a b} \psi^{a b}} H^{t} \partial_{d} H \varepsilon_{c e f d} \sum_{p=1}^{6} F_{n m_{1} \cdots m_{p-1}} S_{g} C \psi^{c e f} \psi^{n m_{1} \cdots m_{p-1}}|0\rangle \\
& +t e^{\frac{1}{2} b_{a b} \psi^{a b}} H^{t-1} \partial_{c} H \sum_{p=1}^{6} F_{n m_{1} \cdots m_{p-1}} S_{g} C \psi^{c} \psi^{n m_{1} \cdots m_{p-1}}|0\rangle
\end{aligned}
$$

We now evaluate the first and the second lines in (B.44) separately. For the first line, since $C \psi^{\mu}=\psi_{\mu} C$, we have

$$
S_{g} C \psi^{c e f} \psi^{n m_{1} \cdots m_{p-1}}|0\rangle=S_{g} \psi_{c e f} \psi_{n m_{1} \cdots m_{p-1}} C|0\rangle .
$$

Further, since

$$
C|0\rangle=\left(\psi^{0}+\psi_{0}\right)\left(\psi^{1}+\psi_{1}\right) \cdots\left(\psi^{9}+\psi_{9}\right)|0\rangle=\psi^{012 \cdots 9}|0\rangle,
$$

by decomposing the indices into the world-volume (012345) and the transverse (6789) directions, we find

$$
\text { (B.45) }=S_{g} \psi_{c e f} \psi^{6789} \psi_{n m_{1} \cdots m_{p-1}} \psi^{012345}|0\rangle \text {. }
$$


Then using the formulae,

$$
\begin{aligned}
\psi_{c e f} \psi^{6789}|0\rangle & =\frac{1}{(4-3) !} \varepsilon_{f e c h} \psi^{h}|0\rangle, \\
\psi_{n m_{1} \cdots m_{p-1}} \psi^{012345}|0\rangle & =\frac{1}{(6-p) !} \varepsilon_{n m_{1} \cdots m_{6-p} k_{1} \cdots k_{6-p}} \psi^{k_{1} \cdots k_{6-p}}|0\rangle,
\end{aligned}
$$

we obtain

$$
(\mathrm{B} .47)=\frac{1}{(6-p) !} \varepsilon_{f e c h} \varepsilon_{n m_{1} \cdots m_{6-p} k_{1} \cdots k_{6-p}} S_{g} \psi^{h} \psi^{k_{1} \cdots k_{6-p}}|0\rangle .
$$

Finally, by using the formula

$$
S_{g} \psi^{\mu_{1} \cdots \mu_{p}}|0\rangle=-\frac{1}{\sqrt{|\operatorname{det} g|}} g_{\mu_{1} \nu_{1}} \cdots g_{\mu_{p} \nu_{p}} \psi^{\nu_{1} \cdots \nu_{p}}|0\rangle
$$

and substituting the explicit solution of the localized DFT monopole, we find

$$
\left(\text { B.49) }=\frac{1}{(6-p) !} \varepsilon_{f e c h} \varepsilon_{n m_{1} \cdots m_{6-p} k_{1} \cdots k_{6-p}}\left(-H^{-2}\right) H \delta^{h d} \eta_{k_{1} l_{1}} \cdots \eta_{k_{6-p} l_{6-p}} \psi^{d} \psi^{l_{1} \cdots l_{6-p}}|0\rangle\right. \text {. }
$$

Likewise, for the second line in (B.44), we have

$$
S_{g} C \psi^{c} \psi^{n m_{1} \cdots m_{p-1}}|0\rangle=-\frac{1}{3 !} \frac{1}{(6-p) !} H \varepsilon_{c e f h} \varepsilon_{n m_{1} \cdots m_{p-1} l_{1} \cdots l_{6-p}} \psi^{e f h} \psi^{l_{1} \cdots l_{6-p}}|0\rangle .
$$

Therefore,

r.h.s. of (B.42)

$$
\begin{aligned}
= & e^{\frac{1}{2} b_{a b} \psi^{a b}}\left[-\frac{1}{6} \frac{1}{(6-p) !} H^{t-1} \partial_{d} H \sum_{p} \varepsilon_{n m_{1} \cdots m_{p-1} k_{1} \cdots k_{6-p}} \eta_{k_{1} l_{1}} \cdots \eta_{k_{6-p} l_{6-p}} F_{n m_{1} \cdots m_{p-1}} \psi^{d^{\prime}} \psi^{l_{1} \cdots l_{6-p}}|0\rangle\right. \\
& \left.-t \frac{1}{3 !} \frac{1}{(6-p) !} H^{t} \partial_{c} H \varepsilon_{c e f h} \sum_{p} \varepsilon_{n m_{1} \cdots m_{p-1} k_{1} \cdots k_{6-p}} \eta_{k_{1} l_{1}} \cdots \eta_{k_{6-p} l_{6-p}} F_{n m_{1} \cdots m_{p-1}} \psi^{e f h} \psi^{l_{1} \cdots l_{p-1}}|0\rangle\right]
\end{aligned}
$$

On the other hand, we evaluate

$$
\begin{aligned}
\text { 1.h.s. of }(\mathrm{B} .42)=e^{\frac{1}{2} b_{a b} \psi^{a b}}[- & t H^{t-1} \partial_{c} H \sum_{p} F_{n m_{1} \cdots m_{p-1}} \psi^{c} \psi^{n m_{1} \cdots m_{p-1}}|0\rangle \\
& \left.-\frac{1}{6} H^{t} \partial_{d} H \varepsilon_{c e f d} \sum_{p} F_{n m_{1} \cdots m_{p-1}} \psi^{c e f} \psi^{n m_{1} \cdots m_{p-1}}|0\rangle\right]
\end{aligned}
$$

Then, by comparing the both sides of (B.42), we obtain the condition

$$
\begin{aligned}
& \sum_{p=0}^{6} \frac{1}{(6-p) !} \varepsilon_{n m_{1} \cdots m_{p-1} k_{1} \cdots k_{6-p}} \eta_{k_{1} l_{1}} \cdots \eta_{k_{6-p} l_{6-p}} F_{n m_{1} \cdots m_{p-1}} \psi^{l_{1} \cdots l_{6-p}}=-t \sum_{p=0}^{6} F_{l_{1} \cdots l_{p}} \psi^{l_{1} \cdots l_{p}}, \\
& \sum_{p=0}^{6} \frac{1}{(6-p) !} \varepsilon_{n m_{1} \cdots m_{p-1} k_{1} \cdots k_{6-p}} \eta_{k_{1} l_{1}} \cdots \eta_{k_{6-p} l_{6-p}} F_{n m_{1} \cdots m_{p-1}} \psi^{l_{1} \cdots l_{6-p}}=-\frac{1}{t} \sum_{p=0}^{6} F_{l_{1} \cdots l_{p}} \psi^{l_{1} \cdots l_{p}} .
\end{aligned}
$$

They are the conditions (4.26). 
Open Access. This article is distributed under the terms of the Creative Commons Attribution License (CC-BY 4.0), which permits any use, distribution and reproduction in any medium, provided the original author(s) and source are credited.

\section{References}

[1] C.M. Hull and P.K. Townsend, Unity of superstring dualities, Nucl. Phys. B 438 (1995) 109 [hep-th/9410167] [INSPIRE].

[2] M. Blau and M. O'Loughlin, Aspects of U duality in matrix theory, Nucl. Phys. B 525 (1998) 182 [hep-th/9712047] [INSPIRE].

[3] N.A. Obers and B. Pioline, U duality and M-theory, Phys. Rept. 318 (1999) 113 [hep-th/9809039] [INSPIRE].

[4] E. Eyras and Y. Lozano, Exotic branes and nonperturbative seven-branes, Nucl. Phys. B $\mathbf{5 7 3}$ (2000) 735 [hep-th/9908094] [INSPIRE].

[5] J. de Boer and M. Shigemori, Exotic branes and non-geometric backgrounds, Phys. Rev. Lett. 104 (2010) 251603 [arXiv: 1004.2521] [INSPIRE].

[6] J. de Boer and M. Shigemori, Exotic Branes in String Theory, Phys. Rept. 532 (2013) 65 [arXiv: 1209.6056] [INSPIRE].

[7] J.J. Fernández-Melgarejo, T. Kimura and Y. Sakatani, Weaving the Exotic Web, JHEP 09 (2018) 072 [arXiv : 1805.12117] [inSPIRE].

[8] D.S. Berman, E.T. Musaev and R. Otsuki, Exotic Branes in Exceptional Field Theory: $E_{7(7)}$ and Beyond, JHEP 12 (2018) 053 [arXiv:1806.00430] [INSPIRE].

[9] T. Kimura and S. Sasaki, Worldsheet Description of Exotic Five-brane with Two Gauged Isometries, JHEP 03 (2014) 128 [arXiv:1310.6163] [INSPIRE].

[10] T. Kimura, S. Sasaki and M. Yata, Hyper-Kähler with torsion, T-duality, and defect $(p, q)$ five-branes, JHEP 03 (2015) 076 [arXiv:1411.3457] [INSPIRE].

[11] T. Kikuchi, T. Okada and Y. Sakatani, Rotating string in doubled geometry with generalized isometries, Phys. Rev. D 86 (2012) 046001 [arXiv:1205.5549] [INSPIRE].

[12] T. Okada and Y. Sakatani, Defect branes as Alice strings, JHEP 03 (2015) 131 [arXiv: 1411.1043] [INSPIRE].

[13] Y. Sakatani, Exotic branes and non-geometric fluxes, JHEP 03 (2015) 135 [arXiv: 1412.8769] [INSPIRE].

[14] F. Hassler and D. Lüst, Non-commutative/non-associative IIA (IIB) Q- and R-branes and their intersections, JHEP 07 (2013) 048 [arXiv:1303.1413] [INSPIRE].

[15] C.M. Hull, A geometry for non-geometric string backgrounds, JHEP 10 (2005) 065 [hep-th/0406102] [INSPIRE].

[16] C. Hull and B. Zwiebach, Double Field Theory, JHEP 09 (2009) 099 [arXiv:0904.4664] [INSPIRE].

[17] W. Siegel, Two vierbein formalism for string inspired axionic gravity, Phys. Rev. D 47 (1993) 5453 [hep-th/9302036] [INSPIRE].

[18] W. Siegel, Superspace duality in low-energy superstrings, Phys. Rev. D 48 (1993) 2826 [hep-th/9305073] [INSPIRE]. 
[19] W. Siegel, Manifest duality in low-energy superstrings, hep-th/9308133 [INSPIRE].

[20] J. Berkeley, D.S. Berman and F.J. Rudolph, Strings and Branes are Waves, JHEP 06 (2014) 006 [arXiv: 1403.7198] [INSPIRE].

[21] D.S. Berman and F.J. Rudolph, Branes are Waves and Monopoles, JHEP 05 (2015) 015 [arXiv: 1409.6314] [INSPIRE].

[22] I. Bakhmatov, A. Kleinschmidt and E.T. Musaev, Non-geometric branes are DFT monopoles, JHEP 10 (2016) 076 [arXiv: 1607.05450] [INSPIRE].

[23] T. Kimura, S. Sasaki and K. Shiozawa, Worldsheet Instanton Corrections to Five-branes and Waves in Double Field Theory, JHEP 07 (2018) 001 [arXiv: 1803.11087] [INSPIRE].

[24] E. Plauschinn, Non-geometric backgrounds in string theory, Phys. Rept. 798 (2019) 1 [arXiv: 1811.11203] [INSPIRE].

[25] D.S. Berman and C.D.A. Blair, The Geometry, Branes and Applications of Exceptional Field Theory, Int. J. Mod. Phys. A 35 (2020) 2030014 [arXiv:2006.09777] [INSPIRE].

[26] R. Gregory, J.A. Harvey and G.W. Moore, Unwinding strings and t duality of Kaluza-Klein and h monopoles, Adv. Theor. Math. Phys. 1 (1997) 283 [hep-th/9708086] [INSPIRE].

[27] D. Tong, NS5-branes, T duality and world sheet instantons, JHEP 07 (2002) 013 [hep-th/0204186] [INSPIRE].

[28] J.A. Harvey and S. Jensen, Worldsheet instanton corrections to the Kaluza-Klein monopole, JHEP 10 (2005) 028 [hep-th/0507204] [INSPIRE].

[29] S. Jensen, The KK-Monopole/NS5-Brane in Doubled Geometry, JHEP 07 (2011) 088 [arXiv:1106.1174] [INSPIRE].

[30] T. Kimura and S. Sasaki, Gauged Linear sigma model for Exotic Five-brane, Nucl. Phys. B 876 (2013) 493 [arXiv: 1304.4061] [INSPIRE].

[31] T. Kimura and S. Sasaki, Worldsheet instanton corrections to $5_{2}^{2}$-brane geometry, JHEP 08 (2013) 126 [arXiv: 1305.4439] [INSPIRE].

[32] T. Kimura, S. Sasaki and K. Shiozawa, Semi-doubled Gauged Linear sigma model for Five-branes of Codimension Two, JHEP 12 (2018) 095 [arXiv: 1810.02169] [INSPIRE].

[33] D. Lüst, E. Plauschinn and V. Vall Camell, Unwinding strings in semi-flatland, JHEP 07 (2017) 027 [arXiv : 1706. 00835] [INSPIRE].

[34] I. Achmed-Zade, J.D. Hamilton, Mark, D. Lüst and S. Massai, A note on T-folds and T ${ }^{3}$ fibrations, JHEP 12 (2018) 020 [arXiv: 1803.00550] [INSPIRE].

[35] A. Chatzistavrakidis, F.F. Gautason, G. Moutsopoulos and M. Zagermann, Effective actions of nongeometric five-branes, Phys. Rev. D 89 (2014) 066004 [arXiv:1309.2653] [INSPIRE].

[36] T. Kimura, S. Sasaki and M. Yata, World-volume Effective Actions of Exotic Five-branes, JHEP 07 (2014) 127 [arXiv: 1404.5442] [INSPIRE].

[37] T. Kimura, S. Sasaki and M. Yata, World-volume Effective Action of Exotic Five-brane in M-theory, JHEP 02 (2016) 168 [arXiv: 1601.05589] [INSPIRE].

[38] Y. Sakatani and S. Uehara, Branes in Extended Spacetime: Brane Worldvolume Theory Based on Duality Symmetry, Phys. Rev. Lett. 117 (2016) 191601 [arXiv:1607.04265] [INSPIRE]. 
[39] C.D.A. Blair and E.T. Musaev, Five-brane actions in double field theory, JHEP 03 (2018) 111 [arXiv: 1712.01739] [INSPIRE].

[40] Y. Sakatani and S. Uehara, Born sigma model for branes in exceptional geometry, PTEP 2020 (2020) 073B05 [arXiv: 2004.09486] [INSPIRE].

[41] O. Hohm, C. Hull and B. Zwiebach, Generalized metric formulation of double field theory, JHEP 08 (2010) 008 [arXiv: 1006.4823] [INSPIRE].

[42] O. Hohm, S.K. Kwak and B. Zwiebach, Unification of Type II Strings and T-duality, Phys. Rev. Lett. 107 (2011) 171603 [arXiv:1106.5452] [INSPIRE].

[43] O. Hohm, S.K. Kwak and B. Zwiebach, Double Field Theory of Type II Strings, JHEP 09 (2011) 013 [arXiv:1107.0008] [INSPIRE].

[44] E.A. Bergshoeff, T. Ortín and F. Riccioni, Defect Branes, Nucl. Phys. B 856 (2012) 210 [arXiv: 1109.4484] [INSPIRE].

[45] T. Adawi, M. Cederwall, U. Gran, B.E.W. Nilsson and B. Razaznejad, Goldstone tensor modes, JHEP 02 (1999) 001 [hep-th/9811145] [INSPIRE].

[46] D.R. Brill and J.B. Hartle, Method of the Self-Consistent Field in General Relativity and its Application to the Gravitational Geon, Phys. Rev. 135 (1964) B271 [INSPIRE].

[47] C.N. Pope, Axial Vector Anomalies and the Index Theorem in Charged Schwarzschild and Taub-NUT Spaces, Nucl. Phys. B 141 (1978) 432 [InSPIRE].

[48] J.-H. Park, Comments on double field theory and diffeomorphisms, JHEP 06 (2013) 098 [arXiv: 1304.5946] [INSPIRE].

[49] K. Lee and J.-H. Park, Covariant action for a string in "doubled yet gauged" spacetime, Nucl. Phys. B $\mathbf{8 8 0}$ (2014) 134 [arXiv:1307.8377] [InSPIRE].

[50] D.S. Berman, C.D.A. Blair and R. Otsuki, Non-Riemannian geometry of M-theory, JHEP 07 (2019) 175 [arXiv: 1902.01867] [inSPIRE].

[51] H. Mori, S. Sasaki and K. Shiozawa, Doubled Aspects of Vaisman Algebroid and Gauge Symmetry in Double Field Theory, J. Math. Phys. 61 (2020) 013505 [arXiv:1901.04777] [INSPIRE].

[52] N. Ikeda and S. Sasaki, Global Aspects of Doubled Geometry and Pre-rackoid, arXiv:2006.08158 [INSPIRE].

[53] H. Mori and S. Sasaki, More on Doubled Aspects of Algebroids in Double Field Theory, J. Math. Phys. 61 (2020) 123504 [arXiv: 2008.00402] [INSPIRE].

[54] O. Hohm and S.K. Kwak, $N=1$ Supersymmetric Double Field Theory, JHEP 03 (2012) 080 [arXiv: 1111.7293] [INSPIRE].

[55] I. Jeon, K. Lee, J.-H. Park and Y. Suh, Stringy Unification of Type IIA and IIB Supergravities under $N=2 D=10$ Supersymmetric Double Field Theory, Phys. Lett. B 723 (2013) 245 [arXiv:1210.5078] [INSPIRE].

[56] C.D.A. Blair and E. Malek, Geometry and fluxes of SL(5) exceptional field theory, JHEP 03 (2015) 144 [arXiv:1412.0635] [INSPIRE].

[57] D.S. Berman and F.J. Rudolph, Strings, Branes and the Self-dual Solutions of Exceptional Field Theory, JHEP 05 (2015) 130 [arXiv:1412.2768] [INSPIRE]. 
[58] M. Günaydin, D. Lüst and E. Malek, Non-associativity in non-geometric string and M-theory backgrounds, the algebra of octonions, and missing momentum modes, JHEP 11 (2016) 027 [arXiv: 1607.06474] [INSPIRE].

[59] D. Lüst, E. Malek and M. Syvari, Locally non-geometric fluxes and missing momenta in M-theory, JHEP 01 (2018) 050 [arXiv:1710.05919] [INSPIRE].

[60] I. Bakhmatov, D. Berman, A. Kleinschmidt, E. Musaev and R. Otsuki, Exotic branes in Exceptional Field Theory: the SL(5) duality group, JHEP 08 (2018) 021 [arXiv:1710.09740] [INSPIRE].

[61] O. Hohm, D. Lüst and B. Zwiebach, The Spacetime of Double Field Theory: Review, Remarks, and Outlook, Fortsch. Phys. 61 (2013) 926 [arXiv:1309.2977] [INSPIRE].

[62] M. Fukuma, T. Oota and H. Tanaka, Comments on T dualities of Ramond-Ramond potentials on tori, Prog. Theor. Phys. 103 (2000) 425 [hep-th/9907132] [INSPIRE]. 